AEI-2013-240

\title{
Non-linear Kaluza-Klein theory for dual fields
}

\author{
Hadi Godazgar, Mahdi Godazgar and Hermann Nicolai \\ Max-Planck-Institut für Gravitationsphysik, \\ Albert-Einstein-Institut, \\ Am Mühlenberg 1, D-14476 Potsdam, Germany \\ Hadi.Godazgar@aei.mpg.de, Mahdi.Godazgar@aei.mpg.de, \\ Hermann. Nicolai@aei.mpg. de
}

October 29,2018

\begin{abstract}
We present non-linear uplift ansätze for all the bosonic degrees of freedom and dual fields in the $S^{7}$ reduction of $D=11$ supergravity to maximal $\mathrm{SO}(8)$ gauged supergravity and test them for the $\mathrm{SO}(7)^{ \pm}$invariant solutions. In particular, we complete the known ansätze for the internal components of the metric and 4 -form flux by constructing a non-linear ansatz for the internal components of the dual 7-form flux. Furthermore, we provide ansätze for the complete set of 56 vector fields, which are given by more general structures than those available from standard Kaluza-Klein theory. The novel features encountered here have no conventional geometric interpretation and provide a new perspective on Kaluza-Klein theory. We study the recently found set of generalised vielbein postulates and, for the $S^{7}$ compactification, we show that they reduce to the $\mathrm{E}_{7(7)}$ Cartan equation of maximal $\mathrm{SO}(8)$ gauged supergravity in four dimensions. The significance of this framework for a higher-dimensional understanding of the embedding tensor and other gauged maximal supergravities is briefly discussed.
\end{abstract}




\section{Introduction}

In this article we continue the investigation of the generalised geometry underlying maximal supergravity on the basis of the $\mathrm{SO}(1,3) \times \mathrm{SU}(8)$ invariant reformulation [1] of $D=11$ supergravity, which has been further developed in very recent work [2, 3, 4, Our main concern here will be the question of whether the consistency results of standard Kaluza-Klein theory can be extended to the 'non-geometrical' structures that arise in this context, and in particular from the vector and form fields and their duals. Focusing on $D=11$ supergravity [5], and more specifically on its $S^{7}$ compactification [6, 7] to maximal gauged $\mathrm{SO}(8)$ supergravity in four dimensions [8] is amply justified by the fact that this theory presents by far the richest structure of all Kaluza-Klein models studied so far, but of course, we expect our results to be relevant also in a more general context.

The question of whether a four-dimensional theory can be obtained by dimensional reduction from higher dimensions, and the question of whether a given compactification of a higher-dimensional theory can be associated with a consistent truncation is clearly an important and pertinent one. Consistency here by definition is taken to mean that any full solution of the lower-dimensional theory should admit an uplift to a full solution of the non-linear higher-dimensional field equations. However, establishing such a relation and its consistency is far from obvious in all but the most trivial examples. In particular, due to the generic emergence of non-abelian gauge theories in Kaluza-Klein compactifications, we have to deal with gauged supergravities. The most efficient framework for understanding these theories (in any dimension) is the embedding tensor formalism [9, 10, 11, 12. Therefore, any general scheme that aims to address the issue of the higher-dimensional origin of four-dimensional theories should provide a higher-dimensional perspective on the embedding tensor. Furthermore, given a consistent truncation, yet another challenging task is to give explicit uplift ansätze for all relevant fields, something that standard Kaluza-Klein theory cannot give for fields other than the graviton and the vector fields.

In [1] a $4+7$ splitting of $D=11$ supergravity is considered with an appropriate decomposition of all eleven-dimensional fields with respect to this splitting, while retaining full on-shell equivalence to the original theory. This reformulation has manifest local SU(8) invariance, and emphasises and generalises the structures that would appear upon a toroidal reduction of the theory to four dimensions [13, 14]. The construction of [1] relies on an analysis of the supersymmetry transformations of the redefined fields and a crucial object that emerges from the supersymmetry transformation of the graviphoton is an $\mathrm{SU}(8)$ tensor, the generalised vielbein. The graviphoton gives rise to vector fields upon reduction. However, in the reduced theory these are complemented by other vector fields. In particular, the three-form potential also contributes to the vector degrees of freedom. The supersymmetry transformation of these vectors in the $D=11$ theory gives rise to yet another generalised vielbein [2]. The observation made in [4] is that by considering dualisation of eleven-dimensional fields, a full set of 56 vectors is obtained whose supersymmetry transformations give rise naturally to an $\mathrm{E}_{7(7)}$ vielbein in eleven dimensions.

The emergence [4 of $\mathrm{E}_{7(7)}$ structures in $D=11$ supergravity gives a new perspective on the extent to which duality symmetries play a role in the full unreduced $D=11$ theory and the necessity to transcend usual notions of geometry. 1 However, the framework that is developed in [4] - based on the $\mathrm{SU}(8)$ invariant reformulation [1] of $D=11$ supergravity and extending the recent results of [2] - is also the most natural setting in which to understand the eleven-dimensional origins of fourdimensional gauged theories. The construction in [4] highlights structures in eleven dimensions that are manifest in the reduced theory, enabling one to address questions concerning uplift ansätze and the appearance of particular gaugings in four dimensions from a reduction point of view.

In this paper, we use the $S^{7}$ reduction to maximal gauged supergravity to illustrate the effec-

\footnotetext{
${ }^{1}$ For alternative approaches to generalised geometry and a list of recent references with bibliographies, see [15].
} 
tiveness of the framework presented in [4]. In particular, we extend the Kaluza-Klein ansätze for the graviphoton [23, 24, 25] and the vector associated with the three-form potential [2] to vectors associated with dual fields, revealing novel features. These ansätze allow us to derive a non-linear ansatz for the internal components of the six-form potential complementing the non-linear ansätze for the internal components of the metric [26] and the three-form potential [2]. A remarkable feature of this analysis is not only the existence of a non-linear ansatz for a dual field, but for all fields.

To illustrate the novelty of the present construction, let us first recall some well-known facts from standard Kaluza-Klein theory [23, 24, 25]. Starting from the higher-dimensional vielbein $E_{M}{ }^{A}(z) \equiv$ $E_{M}^{A}(x, y)$, where the higher-dimensional coordinates $\left\{z^{M}\right\}$ are split into four-dimensional coordinates $\left\{x^{\mu}\right\}$ and internal coordinates $\left\{y^{m}\right\}$, respectively, one proceeds from the ansatz

$$
E_{M}^{A}(x, y)=\left(\begin{array}{cc}
\Delta^{-1 / 2} e_{\mu}^{\prime \alpha} & B_{\mu}^{m} e_{m}^{a} \\
0 & e_{m}{ }^{a}
\end{array}\right),
$$

where $e_{m}{ }^{a}(x, y)$ is the vielbein associated to the internal manifold on which the higher-dimensional theory is assumed to be compactified, $\Delta \equiv \operatorname{det} e_{m}{ }^{a}$ and $e_{\mu}^{\prime \alpha}$ is the Weyl rescaled vierbein of the compactified theory; the triangular form of $E_{M}{ }^{A}$ is arrived at by making partial use of the local Lorentz symmetry of the higher-dimensional theory. A consistent truncation for the spin-two field (graviton) is achieved simply by setting

$$
e_{\mu}^{\alpha}(x, y) \equiv e_{\mu}^{\prime \alpha}(x),
$$

that is, by dropping all dependence on the internal coordinates. Likewise, for the vectors $B_{\mu}{ }^{m}(x, y)$, the exact consistent ansatz has been known for a very long time [23, 24, 25]; it reads

$$
B_{\mu}{ }^{m}(x, y)=K^{m \mathcal{I}}(y) A_{\mu}^{\mathcal{I}}(x),
$$

where the index $\mathcal{I}$ labels the Killing vectors $K^{m \mathcal{I}}(y)$ on the internal manifold. It is a key result of Kaluza-Klein theory that the non-abelian gauge interactions of the compactified theory then originate from the commutator of two Killing vector fields

$$
\left[K^{m \mathcal{I}} \partial_{m}, K^{n \mathcal{J}} \partial_{n}\right]=f^{\mathcal{I} \mathcal{J}}{ }_{\mathcal{K}} K^{p \mathcal{K}} \partial_{p}
$$

where $f^{\mathcal{I} \mathcal{J}} \mathcal{K}$ are the structure constants of the isometry group of the internal manifold. In this way the gauge group of the compactified theory is completely explained in geometric terms. While this has been well understood for many decades, the main difficulty in establishing the full consistency of the Kaluza-Klein reduction resides in the scalar sector, in particular involving the search for consistent ansätze for the internal vielbein $e_{m}{ }^{a}(x, y)$ and other fields of a tensorial nature under internal symmetries. The main focus of the present work, then, is to develop a similar theory for 'non-geometrical' vector fields and matter fields, and in particular for those fields arising from dual fields in higher dimensions, for which no readily applicable formulae are available from general Kaluza-Klein theory-hence the need for a "generalised geometry"!

The structure of the paper is as follows. In section 2, we review the main results of Ref. [4]. We briefly discuss how the consideration of dual fields in eleven dimensions can be used to construct 56 vectors, the supersymmetry transformations of which give rise to a set of generalised vielbeine that are parametrised by the 'internal' components of the eleven-dimensional metric, three-form potential and its dual six-form. The generalised vielbeine can be viewed as components of an $\mathrm{E}_{7(7)}$ matrix and the supersymmetry transformations of the bosonic fields can be cast into a form that mirrors the analogous supersymmetry transformations in four dimensions. Furthermore, the generalised vielbein postulates [1, 4] are summarised in section 2.3. 
Specialising to the $S^{7}$ reduction of $D=11$ supergravity to maximal $\mathrm{SO}(8)$ gauged supergravity in four dimensions in section [3, we use the results summarised in section 2 to derive non-linear ansätze for all bosonic degrees of freedom. In particular, extending the result in Ref. [2, we give Kaluza-Klein ansätze for all 56 vector fields. These ansätze not only include Killing vector fields on $S^{7}$, but also tensors and the potential for the volume form on $S^{7}$, which is not globally defined. Comparing the eleven and four-dimensional supersymmetry transformations of the vectors, along the lines of [26, 2], allows us to express the generalised vielbeine in terms of the four-dimensional scalars. These relations are then used to find a non-linear ansatz for the internal components of the six-form dual potential. The set of ansätze for the vectors and the internal components of the metric, three-form potential and its dual comprise a set of uplift formulae for all bosonic degrees of freedom. In section 5, we test the non-linear ansatz for the 6-form field by explicitly checking that the ansatz reproduces the internal component of the 6 -form potential of the $\mathrm{SO}(7)^{ \pm}$invariant solutions of $D=11$ supergravity [27, 28] from the scalar expectation values of the $\mathrm{SO}(7)^{ \pm}$invariant stationary points [29] of maximal $\mathrm{SO}(8)$ gauged supergravity. The possibility to perform such explicit checks of all formulae against various non-trivial compactifications is a feature that distinguishes our formalism from other approaches to generalised geometry.

From a four-dimensional point of view, the 56 vector fields include the full set of electric and magnetic vectors that can be gauged. In section 4 , we show that the generalised vielbein postulates determine exactly which of the vector fields are gauged in the $S^{7}$ compactification. In particular, we show that upon inserting the ansätze relevant for the $S^{7}$ compactification given in section 3 , the magnetic vector fields, which come from the reduction of the 3 -form potential, drop out of the expressions. Moreover, the generalised vielbein postulates reduce to the $\mathrm{E}_{7(7)}$ Cartan equation with $\mathrm{SO}(8)$ gauge covariant derivative. The $\mathrm{SO}(8)$ gauge fields are solely electric and arise from the graviphoton and the 6-form potential. More generally, for any compactification, the generalised vielbein postulates reduce to the four-dimensional Cartan equation with the appropriate gauge covariant derivative. Therefore, the generalised vielbein postulates provide an understanding of how the gauge vectors are selected from the 56 vector fields available. This goes some way towards establishing the origin of the embedding tensor in the higher-dimensional $D=11$ theory.

We conclude in section 6 with a brief, general discussion of other compactifications that could lead to more general gaugings in four dimensions. Of particular interest are examples of compactifications where both electric and magnetic vectors are gauged. We also discuss the possibility of using our framework to provide an eleven-dimensional perspective on the recently discovered continuous family of $\mathrm{SO}(8)$ gauged supergravities 30 .

In summary, the key results and issues raised in this paper are:

- Kaluza-Klein theory is developed for 'non-geometric' vector fields.

- Consistent non-linear ansätze are obtained for all fields, including dual fields.

- All formulae can be tested against non-trivial compactifications of $D=11$ supergravity and the associated stationary points of $N=8$ supergravity.

- With 56 'electric' and 'magnetic' vectors present in all $D=11$ relations, one can now study the higher-dimensional origins of the embedding tensor.

- Preliminary evidence is presented that the $\omega$-deformed $\mathrm{SO}(8)$ gaugings of [30] correspond to $\omega$-deformations of $D=11$ supergravity.

The conventions and index notations used in this paper are as in [1, 4]. 


\section{Dual fields and $\mathbf{E}_{7(7)}$ in $D=11$ supergravity}

In Ref. [4], the two generalised vielbeine previously known from the literature [1, 2], are completed to an $\mathrm{E}_{7(7)}$ matrix in eleven dimensions by constructing two further generalised vielbeine that are intimately related to the dualisation of the metric and the three-form potential in eleven dimensions. While the significance of this construction from an eleven-dimensional point of view is clear in that it establishes the role of the $\mathrm{E}_{7(7)}$ duality group in the full $D=11$ theory, its importance from the practical point of view of relating the four-dimensional maximal supergravity to $D=11$ supergravity is what will be addressed in this paper. In particular, the results of [4] give uplift ansätze for all bosonic degrees of freedom including a non-linear ansatz for the six-form potential dual to the three-form potential. We will illustrate this in great detail for the $S^{7}$ compactification of $D=11$ supergravity in the following sections, but from the generality of our results it should be clear that our construction furnishes similar information for other compactifications of $D=11$ supergravity. Consequently, we will first summarise the general results in this section, without reference to any specific compactification.

\subsection{Dual vector fields and generalised vielbeine}

Working in the context of the $\mathrm{SU}(8)$ invariant reformulation of $D=11$ supergravity one identifies certain $\mathrm{SU}(8)$ objects starting from an analysis of the fermionic sector [1]. 2] In the bosonic sector the most prominent of these objects are the so-called generalised vielbeine, which can be regarded as components of an $\mathrm{E}_{7(7)}$ matrix in eleven dimensions. The generalised vielbeine appear when one considers the supersymmetry transformation of those components of the elfbein, three-form potential and their dual fields which in a proper reduction to four dimensions would give rise to vector fields. However, it is important to keep in mind the main feature of the present analysis (and of [1]), namely that we retain the full coordinate dependence on all eleven coordinates throughout. Therefore, we will not be dealing with a dimensional reduction in the strict sense of the word, but rather a $4+7$ split and a subsequent reformulation of the theory. Furthermore, the reformulated theory is on-shell equivalent to the original $D=11$ supergravity of [5] at all stages of the construction.

Let us therefore first consider the spin-one sector of the theory. In the direct dimensional reduction of $D=11$ supergravity to four dimensions there appear only 28 vector fields, namely [14]

$$
B_{\mu}{ }^{m} \quad \text { and } \quad B_{\mu m n}=A_{\mu m n}-B_{\mu}^{p} A_{p m n} .
$$

The first seven of these are just the standard Kaluza-Klein vector fields in the decomposition of the elfbein displayed in (11), while the second set of vectors originates from the three-form field $A_{M N P}$. As explained in our previous work [4], this set of vector fields is complemented by another set of vectors related to the dual fields in eleven dimensions, viz. 3

$$
B_{\mu m_{1} \ldots m_{5}}=A_{\mu m_{1} \ldots m_{5}}-B_{\mu}^{p} A_{p m_{1} \ldots m_{5}}-\frac{\sqrt{2}}{4}\left(A_{\mu\left[m_{1} m_{2}\right.}-B_{\mu}^{p} A_{p\left[m_{1} m_{2}\right.}\right) A_{\left.m_{3} m_{4} m_{5}\right]}
$$

\footnotetext{
${ }^{2}$ See also section 3.1 of Ref. [4] for a brief description of the SU(8) invariant reformulation.

${ }^{3}$ Note the slight change in notation here compared with that used in 4 . In particular, here, we reserve the notation $B_{\mu m_{1} \ldots m_{5}}$ for the vector whose supersymmetry transformation gives rise to the generalised vielbein $e_{m_{1} \ldots m_{5} A B}$. A similar change of notation is made for the fourth vector. In addition, the arbitrary constant $\tilde{c}$ here is related to $c$ in [4] by $\tilde{c}=5 ! c / \sqrt{2}$.
} 


$$
\begin{aligned}
& B_{\mu m_{1} \ldots m_{7}, n}=A_{\mu m_{1} \ldots m_{7}, n}+(3 \tilde{c}-1)\left(A_{\mu\left[m_{1} \ldots m_{5}\right.}-B_{\mu}{ }^{p} A_{p\left[m_{1} \ldots m_{5}\right.}\right) A_{\left.m_{6} m_{7}\right] n} \\
&+\tilde{c} A_{\left[m_{1} \ldots m_{6}\right.}\left(A_{\left.|\mu| m_{7}\right] n}-B_{\mu}{ }^{p} A_{\left.|p| m_{7}\right] n}\right) \\
&+\frac{\sqrt{2}}{12}\left(A_{\mu\left[m_{1} m_{2}\right.}-B_{\mu}{ }^{p} A_{p\left[m_{1} m_{2}\right.}\right) A_{m_{3} m_{4} m_{5}} A_{\left.m_{6} m_{7}\right] n} .
\end{aligned}
$$

The vector components in (5) thus originate from the six-form $A_{(6)}$ which is the dual potential associated with the three-form potential $A_{(3)}$, whence it is clear that these fields are simultaneously defined only on-shell, as explained in [4]. The vector fields (6) are related to the dual gravity field in eleven dimensions, and are defined only up to a real constant $\tilde{c}$. While the precise relation of $A_{\mu m_{1} \ldots m_{7}, n}$ to the $D=11$ fields is not known, the indeterminacy encoded in the parameter $\tilde{c}$ can be traced back to the fact that dual gravity does not give rise to scalar degrees of freedom in the $4+7$ split. We also note that the non-linear modifications in these equations which involve the Kaluza-Klein vectors $B_{\mu}{ }^{m}$ can be understood geometrically via the conversion of curved to flat indices, whereas the remaining non-linear modifications are required by the consistency of the supersymmetry variations, but have no direct explanation in terms of eleven-dimensional geometry. 4

Thus, in all we have identified 56 such vector fields in eleven dimensions, starting from the fields of $D=11$ supergravity and their duals. These make up part of the bosonic sector of our reformulation of $D=11$ supergravity in the framework of the "generalised geometry" introduced in [4. However, not all of these vector fields will correspond to independent propagating vectors in a given compactification of the $D=11$ theory. In particular, for compactifications related to $N=8$ supergravity and deformations thereof, we know that there can be at most 28 propagating spin-one degrees of freedom. This is most easily seen in the $T^{7}$ reduction of [14], where the seven 'electric' vectors from $B_{\mu}{ }^{m}$ (corresponding to the seven Killing vectors on $T^{7}$ ) combine with 21 'magnetic' vectors from $B_{\mu m n}$ to give 28 abelian vector fields. The other 28 vectors correspond to their four-dimensional duals such that the eleven-dimensional duality relations reduce to the 'twisted self duality constraint' of [14] in the reduction to four dimensions. For non-trivial compactifications of the theory the situation is, however, much more complicated because of the appearance of nonabelian gauge interactions, for which the usual (abelian) dualisation of vector fields does not work.

A judicious analysis of the supersymmetry transformations of these 56 vector fields [1, 2, 4, leads to the generalised vielbeine. For the vector fields (44) the latter can be directly obtained from the $D=11$ theory, while the variation of $B_{\mu m_{1} \ldots m_{5}}$ in (5) is determined from the variation of $A_{(6)}$ [4. The supersymmetry transformation of $B_{\mu m_{1} \ldots m_{7}, n}$ is also given in Ref. [4], but it cannot be obtained from the $D=11$ theory. It can, however, be obtained by imposing consistency with the supersymmetry variations of the other vector fields. Somewhat lengthy computations show that [4]

$$
\begin{aligned}
\delta B_{\mu}^{m} & =\frac{\sqrt{2}}{8} e_{A B}^{m}\left[2 \sqrt{2} \bar{\varepsilon}^{A} \varphi_{\mu}^{B}+\bar{\varepsilon}_{C} \gamma_{\mu}^{\prime} \chi^{A B C}\right]+\text { h.c., } \\
\delta B_{\mu m n} & =\frac{\sqrt{2}}{8} e_{m n A B}\left[2 \sqrt{2} \bar{\varepsilon}^{A} \varphi_{\mu}^{B}+\bar{\varepsilon}_{C} \gamma_{\mu}^{\prime} \chi^{A B C}\right]+\text { h.c., } \\
\delta B_{\mu m_{1} \ldots m_{5}} & =\frac{\sqrt{2}}{8} e_{m_{1} \ldots m_{5} A B}\left[2 \sqrt{2} \bar{\varepsilon}^{A} \varphi_{\mu}^{B}+\bar{\varepsilon}_{C} \gamma_{\mu}^{\prime} \chi^{A B C}\right]+\text { h.c., } \\
\delta B_{\mu m_{1} \ldots m_{7}, n} & =\frac{\sqrt{2}}{8} e_{m_{1} \ldots m_{7}, n A B}\left[2 \sqrt{2} \bar{\varepsilon}^{A} \varphi_{\mu}^{B}+\bar{\varepsilon}_{C} \gamma_{\mu}^{\prime} \chi^{A B C}\right]+\text { h.c. }
\end{aligned}
$$

\footnotetext{
${ }^{4} \mathrm{~A}$ geometrical explanation might, however, follow from $\mathrm{E}_{10}$ where the 'vielbein' comprises not only the gravitational, but also the three- and six-form fields.
} 
where $\varphi_{\mu}^{A}$ and $\chi^{A B C}$ are the (chiral) fermions in the $\mathrm{SU}(8)$ invariant reformulation, and where $\gamma_{\mu}^{\prime}=e_{\mu}^{\alpha}{ }^{\alpha} \gamma_{\alpha}$ with the Weyl rescaled vierbein $e_{\mu}^{\alpha}$ from (11). The scalar coefficients in front of the fermionic bilinears make up the generalised vielbeine, and are given by [1, 2, 4]

$$
\begin{aligned}
& e_{A B}^{m}= i \Delta^{-1 / 2} \Gamma_{A B}^{m} \\
& e_{m n A B}=-\frac{\sqrt{2}}{12} i \Delta^{-1 / 2}\left(\Gamma_{m n A B}+6 \sqrt{2} A_{m n p} \Gamma_{A B}^{p}\right) \\
& e_{m_{1} \ldots m_{5} A B}=\frac{1}{6 ! \sqrt{2}} i \Delta^{-1 / 2}\left[\Gamma_{m_{1} \ldots m_{5} A B}+60 \sqrt{2} A_{\left[m_{1} m_{2} m_{3}\right.} \Gamma_{\left.m_{4} m_{5}\right] A B}\right.\left.-6 ! \sqrt{2}\left(A_{p m_{1} \ldots m_{5}}-\frac{\sqrt{2}}{4} A_{p\left[m_{1} m_{2}\right.} A_{\left.m_{3} m_{4} m_{5}\right]}\right) \Gamma_{A B}^{p}\right], \\
& e_{m_{1} \ldots m_{7}, n A B}=-\frac{2}{9 !} i \Delta^{-1 / 2}\left[\left(\Gamma_{m_{1} \ldots m_{7}} \Gamma_{n}\right) A B\right.+126 \sqrt{2} A_{n\left[m_{1} m_{2}\right.} \Gamma_{\left.m_{3} \ldots m_{7}\right] A B} \\
&+3 \sqrt{2} \times 7 !\left(A_{n\left[m_{1} \ldots m_{5}\right.}+\frac{\sqrt{2}}{4} A_{n\left[m_{1} m_{2}\right.} A_{m_{3} m_{4} m_{5}}\right) \Gamma_{\left.m_{6} m_{7}\right] A B} \\
&\left.+\frac{9 !}{2}\left(A_{n\left[m_{1} \ldots m_{5}\right.}+\frac{\sqrt{2}}{12} A_{n\left[m_{1} m_{2}\right.} A_{m_{3} m_{4} m_{5}}\right) A_{\left.m_{6} m_{7}\right] p} \Gamma_{A B}^{p}\right] .
\end{aligned}
$$

These objects carry $\mathrm{SU}(8)$ indices $A, B, \ldots$ and are to be regarded as $\mathrm{SU}(8)$ tensors in a specific gauge, as explained in [1]. As also shown there, one thereby enlarges the original tangent space symmetry from $\mathrm{SO}(7)$ to a local $\mathrm{SU}(8)$ symmetry that acts on the chiral fermions. Observe that the new vielbein components, $e_{m_{1} \ldots m_{5} A B}$ and $e_{m_{1} \ldots m_{7}, n A B}$, which originate from the variations of the dual vectors (5) and (6), themselves depend on the dual six-form field $A_{m_{1} \ldots m_{6}}$, and hence again are only defined on-shell.

\section{$2.2 \quad$ Emergence of $\mathrm{E}_{7(7)}$ structure}

One can make the relation of the above expressions to $\mathrm{E}_{7(7)}$ more explicit by combining the matrix blocks into a single 56-bein in eleven dimensions [4]

$$
\mathcal{V}(z)=\left(\mathcal{V}^{\mathrm{MN}}{ }_{A B}(z), \mathcal{V}_{\mathrm{MN}} A B(z)\right)
$$

by means of the identifications

$$
\begin{array}{ll}
\mathcal{V}_{A B}^{m 8}=\frac{\sqrt{2}}{8} e_{A B}^{m}, & \mathcal{V}_{m n A B}=-\frac{3}{2} e_{m n A B}, \\
\mathcal{V}_{A B}^{m n}=-\frac{3}{2} \Delta \epsilon^{m n p_{1} \ldots p_{5}} e_{p_{1} \ldots p_{5} A B}, & \mathcal{V}_{m 8 A B}=\frac{9 \sqrt{2}}{2} \Delta \epsilon^{n_{1} \ldots n_{7}} e_{n_{1} \ldots n_{7}, m A B} .
\end{array}
$$

This $\mathrm{E}_{7(7)}$ vielbein is equivalent (see [4]) to the one considered in Ref. [31] in the context of another proposal to realise an exceptional geometry. Note that complex conjugation acts by raising or lowering the $\mathrm{SU}(8)$ indices, viz.

$$
\left(\mathcal{V}_{A B}^{\mathrm{MN}}\right)^{*}=\mathcal{V}^{\mathrm{MN} A B}, \quad\left(\mathcal{V}_{\mathrm{MN}}{ }^{A B}\right)^{*}=\mathcal{V}_{\mathrm{MN}} A B,
$$


but leaves the position of the $\mathrm{SL}(8, \mathbb{R})$ indices $\mathrm{M}, \mathrm{N}$ unaffected. The 56-bein $\mathcal{V}(z)$ as defined above is a coset element of $\mathrm{E}_{7(7)} / \mathrm{SU}(8)$ written in terms of the decomposition of the $\mathbf{5 6}$ of $\mathrm{E}_{7(7)}$ under initially its $\operatorname{SL}(8, \mathbb{R})$ and then $\mathrm{GL}(7, \mathbb{R})$ subgroups

$$
56 \rightarrow \mathbf{2 8} \oplus \overline{\mathbf{2 8}} \rightarrow \mathbf{7} \oplus \mathbf{2 1} \oplus \overline{\mathbf{2 1}} \oplus \overline{\mathbf{7}} .
$$

The world indices $m, n, \ldots$ labeling the seven-dimensional directions and originally transforming under seven-dimensional diffeomorphisms thus become associated with the GL( $7, \mathbb{R})$ subgroup of $\mathrm{E}_{7(7)}$. In contrast to [4] we have adjusted the normalisation of the matrix blocks in such a way that $\mathcal{V}(z)$, as defined in (16) satisfies the following identity

$$
\mathcal{V}_{\mathrm{MN} A B} \mathcal{V}^{\mathrm{MN} C D}-\mathcal{V}^{\mathrm{MN}}{ }_{A B} \mathcal{V}_{\mathrm{MN}}{ }^{C D}=i \delta_{A B}^{C D} \text {. }
$$

This is simply the statement that the inverse of an $\mathrm{E}_{7(7)}$ matrix is related to its complex conjugate; more specifically, (19) is a necessary condition expressing the fact that any $\mathrm{E}_{7(7)}$ matrix automatically belongs to $\operatorname{Sp}(56, \mathbb{R})[14,8]$. The direct verification of (19) by substitution of (11)-(14) into (16) is straightforward.

The vectors can be arranged into a similar object of the form $\left(\mathcal{B}_{\mu}{ }^{\mathrm{MN}}, \mathcal{B}_{\mu \mathrm{MN}}\right)$ such that

$$
\begin{aligned}
\mathcal{B}_{\mu}{ }^{m 8} & =-\frac{1}{2} B_{\mu}{ }^{m}, & \mathcal{B}_{\mu m n} & =3 \sqrt{2} B_{\mu m n}, \\
\mathcal{B}_{\mu}{ }^{m n} & =3 \sqrt{2} \Delta \epsilon^{m n p_{1} \ldots p_{5}} B_{\mu p_{1} \ldots p_{5}}, & \mathcal{B}_{\mu m 8} & =-18 \Delta \epsilon^{n_{1} \ldots n_{7}} B_{\mu n_{1} \ldots n_{7}, m} .
\end{aligned}
$$

Thereby the $28+28$ vectors are combined into a $\mathbf{5 6}$ of $\mathrm{E}_{7(7)}$. In this language, the supersymmetry transformations (7)-(10) can be compactly written as

$$
\begin{aligned}
& \delta \mathcal{B}_{\mu}{ }^{\mathrm{MN}}=-\frac{1}{2} \mathcal{V}^{\mathrm{MN}}{ }_{A B}\left[2 \sqrt{2} \bar{\varepsilon}^{A} \varphi_{\mu}^{B}+\bar{\varepsilon}_{C} \gamma_{\mu}^{\prime} \chi^{A B C}\right]+\text { h.c. } \\
& \delta \mathcal{B}_{\mu \mathrm{MN}}=-\frac{1}{2} \mathcal{V}_{\mathrm{MN} A B}\left[2 \sqrt{2} \bar{\varepsilon}^{A} \varphi_{\mu}^{B}+\bar{\varepsilon}_{C} \gamma_{\mu}^{\prime} \chi^{A B C}\right]+\text { h.c. }
\end{aligned}
$$

In [4] we have also shown that the matrix blocks making up the 56-bein (15) transform uniformly under local supersymmetry

$$
\delta \mathcal{V}^{\mathrm{MN}}{ }_{A B}(z)=-\sqrt{2} \Sigma_{A B C D} \mathcal{V}^{\mathrm{MN} C D}(z), \quad \delta \mathcal{V}_{\mathrm{MN} A B}(z)=-\sqrt{2} \Sigma_{A B C D} \mathcal{V}_{\mathrm{MN}}{ }^{C D}(z)
$$

with the complex self-dual tensor

$$
\Sigma_{A B C D} \equiv \bar{\varepsilon}_{[A} \chi_{B C D]}+\frac{1}{24} \epsilon_{A B C D E F G H} \bar{\varepsilon}^{E} \chi^{F G H}
$$

where we have discarded a local $\mathrm{SU}(8)$ rotation that also acts uniformly on all components. In the form (21) and (22) the supersymmetry variations of compactified maximal supergravity can be read off directly from the $D=11$ formulae.

\subsection{Generalised vielbein postulates}

The generalised vielbeine satisfy certain differential constraints derived in [1, 4]. These constraints are generalisations of the vielbein postulate in Riemannian geometry which establishes the relation 
between affine and spin connections. For our subsequent analysis, here we only need their components along the four space-time directions, which are [1, 4]

$$
\begin{aligned}
& \mathcal{D}_{\mu} e_{A B}^{m}+\frac{1}{2} \partial_{n} B_{\mu}{ }^{n} e_{A B}^{m}+\partial_{n} B_{\mu}{ }^{m} e_{A B}^{n}+\mathcal{Q}_{\mu}^{C}\left[A e_{B] C}^{m}+\mathcal{P}_{\mu A B C D} e^{m C D}=0,\right. \\
& \mathcal{D}_{\mu} e_{m n A B}+\frac{1}{2} \partial_{p} B_{\mu}^{p} e_{m n A B}+2 \partial_{[m} B_{|\mu|}^{p} e_{n] p A B}+3 \partial_{[m} B_{|\mu| n p]} e_{A B}^{p} \\
& +\mathcal{Q}_{\mu[A}^{C} e_{m n B] C}+\mathcal{P}_{\mu A B C D} e_{m n}{ }^{C D}=0, \\
& \mathcal{D}_{\mu} e_{m_{1} \ldots m_{5} A B}+\frac{1}{2} \partial_{p} B_{\mu}{ }^{p} e_{m_{1} \ldots m_{5} A B}-5 \partial_{\left[m_{1}\right.} B_{|\mu|}{ }^{p} e_{\left.m_{2} \ldots m_{5}\right] p A B}+\frac{3}{\sqrt{2}} \partial_{\left[m_{1}\right.} B_{|\mu| m_{2} m_{3}} e_{\left.m_{4} m_{5}\right] A B} \\
& -6 \partial_{\left[m_{1}\right.} B_{\left.|\mu| m_{2} \ldots m_{5} p\right]} e_{A B}^{p}+\mathcal{Q}_{\mu[A}^{C} e_{\left.m_{1} \ldots m_{5} B\right] C}+\mathcal{P}_{\mu A B C D} e_{m_{1} \ldots m_{5}}{ }^{C D}=0, \\
& \mathcal{D}_{\mu} e_{m_{1} \ldots m_{7}, n A B}-\frac{1}{2} \partial_{p} B_{\mu}^{p} e_{m_{1} \ldots m_{7}, n A B}-\partial_{n} B_{\mu}^{p} e_{m_{1} \ldots m_{7}, p A B}+5 \partial_{\left[m_{1}\right.} B_{|\mu| m_{2} m_{3}} e_{\left.m_{4} \ldots m_{7}\right] n A B} \\
& -2 \partial_{\left[m_{1}\right.} B_{|\mu| m_{2} \ldots m_{6}} e_{\left.m_{7}\right] n A B}+\mathcal{Q}_{\mu[A}^{C} e_{\left.m_{1} \ldots m_{7}, n B\right] C}+\mathcal{P}_{\mu A B C D} e_{m_{1} \ldots m_{7}, n}{ }^{C D}=0,
\end{aligned}
$$

where 5

$$
\mathcal{D}_{\mu} \equiv \partial_{\mu}-B_{\mu}^{m} \partial_{m}
$$

In comparison with equation (88) of [4] the generalised vielbein postulate for $e_{m_{1} \ldots m_{7}, n A B}$ has been simplified by using the Schouten identity over eight indices. The connection coefficients $Q_{\mu}$ and $P_{\mu}$ appearing in these equations are valued in the $\mathrm{E}_{7(7)}$ Lie algebra, and are related to the connections and four-form field strengths of $D=11$ supergravity as follows [1]

$$
\begin{aligned}
& \mathcal{Q}_{\mu B}^{A}=-\frac{1}{2}\left[e^{m}{ }_{a} \partial_{m} B_{\mu}^{n} e_{n b}-\left(e^{p}{ }_{a} \mathcal{D}_{\mu} e_{p b}\right)\right] \Gamma_{A B}^{a b}-\frac{\sqrt{2}}{12} \Delta^{-1 / 2} e_{\mu}^{\prime \alpha}\left(F_{\alpha a b c} \Gamma_{A B}^{a b c}-\eta_{\alpha \beta \gamma \delta} F^{\beta \gamma \delta a} \Gamma_{a A B}\right), \\
& \mathcal{P}_{\mu A B C D}=\frac{3}{4}\left[e^{m}{ }_{a} \partial_{m} B_{\mu}{ }^{n} e_{n b}-\left(e^{p}{ }_{a} \mathcal{D}_{\mu} e_{p b}\right)\right] \Gamma_{[A B}^{a} \Gamma_{C D]}^{b}-\frac{\sqrt{2}}{8} \Delta^{-1 / 2} e_{\mu}^{\prime \alpha} F_{a b c \alpha} \Gamma_{[A B}^{a} \Gamma_{C D]}^{b c} \\
& -\frac{\sqrt{2}}{48} \Delta^{-1 / 2} e_{\mu \alpha}^{\prime} \eta^{\alpha \beta \gamma \delta} F_{a \beta \gamma \delta} \Gamma_{b[A B} \Gamma_{C D]}^{a b} .
\end{aligned}
$$

Below we will use them in a slightly modified form, again adapted to the $S^{7}$ compactification.

\section{Non-linear ansätze for maximal supergravity on $S^{7}$}

We will now illustrate the usefulness of the results in the foregoing section by specialising to the $S^{7}$ compactification of $D=11$ supergravity [6, 7], where our formalism furnishes numerous new insights and results, most notably with regard to the dual fields of $D=11$ supergravity. To this aim, we will present a detailed analysis of the non-linear ansätze and the generalised vielbein postulate in the context of the $S^{7}$ compactification.

\subsection{Compactification on $S^{7}$}

To begin with, and for the reader's convenience, here we collect some relevant (and well-known) formulae, see e.g. [7, 3], related to the seven-sphere. Denoting the $S^{7}$ background covariant derivative

\footnotetext{
${ }^{5}$ Below we will work with a slightly modified operator $\mathcal{D}_{\mu}$ adapted to the $S^{7}$ compactification, cf. (59).
} 
by $\stackrel{\circ}{D}_{m}$, we recall that $S^{7}$ admits eight Killing spinors $\eta^{I}(y)$ obeying

$$
\left(\stackrel{\circ}{D}_{m}+\frac{1}{2} i m_{7} \stackrel{\circ}{e}_{m}^{a} \Gamma_{a}\right) \eta^{I}=0
$$

where $I, J, \ldots=1, \ldots, 8$, and $\stackrel{\circ}{m}_{m}^{a}$ is the siebenbein on the round $S^{7}$. Written out in components, the Killing spinors $\eta_{A}^{I}$ are orthonormal matrices, that is

$$
\eta_{I}^{A} \eta_{A}^{J}=\delta_{J}^{I}, \quad \eta_{I}^{A} \eta_{B}^{I}=\delta_{B}^{A}
$$

The 28 Killing vectors $K^{m I J}(y)$ and their derivatives $K_{m n}{ }^{I J}(y)$ can then be represented as bilinears in terms of Killing spinors, viz.

$$
K^{m I J}=i e^{\circ a} \bar{\eta}^{I} \Gamma_{a} \eta^{J}, \quad K_{m n}^{I J}=\stackrel{\circ}{e}_{m}{ }^{a} \stackrel{\circ}{e}_{n}^{b} \bar{\eta}^{I} \Gamma_{a b} \eta^{J}
$$

clearly,

$$
\stackrel{\circ}{D}_{n} K_{m}^{I J}=m_{7} K_{m n}^{I J} \Rightarrow \stackrel{\circ}{D}_{m} K_{n}^{I J}+\stackrel{\circ}{D}_{n} K_{m}^{I J}=0 .
$$

Hence, $\stackrel{\circ}{D}_{n} K^{n I J}=0$. Observe the different 'canonical' positions of the world indices on $K^{m}$ and $K_{m n}$, and the fact that both of these are always and by definition related to the 'flat' objects by means of the $S^{7}$ background siebenbein and its inverse. The vector fields $K^{I J} \equiv K^{m I J} \stackrel{\circ}{D}_{m}$ generate the $\mathrm{SO}(8)$ isometry group of the seven-sphere via the Lie bracket

$$
\left[K^{I J}, K^{K L}\right]=-8 m_{7} \delta^{[I \mid[K} K^{L] \mid J]}
$$

or equivalently

$$
K^{n I J} \stackrel{\circ}{D}_{n} K^{m K L}-K^{n K L} \stackrel{\circ}{D}_{n} K^{m I J}=-8 m_{7} \delta^{[I \mid[K} K^{m L] \mid J]} .
$$

However, in standard Kaluza-Klein geometry there is no corresponding interpretation for the tensor fields $K_{m n}^{I J}$ (nor for (47) below).

\subsection{The non-linear ansätze}

The non-linear ansätze for maximal supergravity are obtained by comparing the supersymmetry transformations (21), with the analogous supersymmetry transformations of the vectors in $D=4$ maximal supergravity [8, 12]:

$$
\begin{aligned}
& \delta A_{\mu}{ }^{I J}=-\frac{1}{2}\left(u_{i j}{ }^{I J}+v_{i j I J}\right)\left[2 \sqrt{2} \bar{\varepsilon}^{i} \varphi_{\mu}^{j}+\bar{\varepsilon}_{k} \gamma_{\mu}^{\prime} \chi^{i j k}\right]+\text { h.c. } \\
& \delta A_{\mu I J}=-\frac{1}{2} i\left(u_{i j}{ }^{I J}-v_{i j I J}\right)\left[2 \sqrt{2} \bar{\varepsilon}^{i} \varphi_{\mu}^{j}+\bar{\varepsilon}_{k} \gamma_{\mu}^{\prime} \chi^{i j k}\right]+\text { h.c. }
\end{aligned}
$$

We will refer to the $28+28$ vector fields $A_{\mu}^{I J}$ and $A_{\mu I J}$ as 'electric' and 'magnetic' vectors, respectively.

In order to relate the $D=4$ vectors to the vector fields identified in the previous section, we now need to choose appropriate Kaluza-Klein ansätze for all vectors (41), (5) and (6). For the Kaluza-Klein vector $B_{\mu}{ }^{m}$ this ansatz is well-known, as we explained in the introduction; choosing appropriate normalisations, we have

$$
B_{\mu}^{m}(x, y)=-\frac{\sqrt{2}}{4} K^{m I J}(y) A_{\mu}^{I J}(x)
$$


where $K^{m I J}$ are the 28 Killing vectors defined in equation (33). However, for the remaining vector fields, and in particular for those arising from the dual fields in higher dimensions, there are no such ansätze available from general Kaluza-Klein theory, and therefore we have to proceed in a different manner. In fact, for the 'non-geometrical' vector fields $B_{\mu m n}(x, y)$ the appropriate ansatz was already found in $[2$; it reads 6

$$
B_{\mu m n}(x, y)=\frac{1}{24} K_{m n}^{I J}(y) A_{\mu I J}(x) .
$$

We stress that the 'canonical' position of the world indices as defined in Ref. 22 is in accord with the position of indices on $B_{\mu}{ }^{m}$ and $B_{\mu m n}$. We emphasise again that, unlike for the standard KaluzaKlein vector, there is a priori no geometric argument to fix the ansatz for $B_{\mu m n}$. Furthermore, the normalisation had to be determined in [3] by comparison with the $D=4$ theory.

Adopting the Kaluza-Klein ansätze (39) and (40) for $B_{\mu}{ }^{m}$ and $B_{\mu m n}$, respectively, and comparing the eleven-dimensional supersymmetry transformations (7) and (8) with the respective fourdimensional transformations (37) and (38) gives a relation between the generalised vielbeine $e_{A B}^{m}$ and $e_{m n A B}$ and the four-dimensional scalars $u_{i j}^{I J}$ and $v_{i j I J}$. More precisely, the identification between 11-dimensional and 4-dimensional $\mathrm{SU}(8)$ indices is made by means of the orthonormal Killing spinors on the round sphere $\eta_{A}^{i}$, which convert 'curved' $\mathrm{SU}(8)$ indices $A, B, \ldots$ (appropriate to the $D=11$ theory) into 'flat' $\mathrm{SU}(8)$ indices $i, j, k, \ldots$ (appropriate to maximal $D=4$ gauged supergravity) and vice versa in the terminology of [32]. Hence,

$$
X_{i j k \cdots}=\eta_{i}^{A} \eta_{j}^{B} \eta_{k}^{C} \cdots X_{A B C \cdots} \quad \Leftrightarrow \quad X_{A B C \cdots}=\eta_{A}^{i} \eta_{B}^{j} \eta_{C}^{k} \cdots X_{i j k \cdots}
$$

for any SU(8) tensor by orthonormality of the Killing spinors (32). Accordingly, we define

$$
\begin{aligned}
e_{i j}^{m}(x, y) & \equiv e_{A B}^{m}(x, y) \eta_{i}{ }^{A}(y) \eta_{j}{ }^{B}(y) \\
e_{m n i j}(x, y) & \equiv e_{m n A B}(x, y) \eta_{i}{ }^{A}(y) \eta_{j}{ }^{B}(y) .
\end{aligned}
$$

The non-linear ansätze derived in previous work are then given by

$$
\begin{aligned}
e_{i j}^{m}(x, y) & =K^{m I J}(y)\left[u_{i j}{ }^{I J}+v_{i j I J}\right](x), \\
e_{m n i j}(x, y) & =-\frac{\sqrt{2}}{12} i K_{m n}^{I J}(y)\left[u_{i j}{ }^{I J}-v_{i j I J}\right](x) .
\end{aligned}
$$

The ansätze for the remaining vectors originating from the dual $D=11$ fields are more tricky and, in fact, can only be arrived at by imposing consistency of the relevant supersymmetry variations. More specifically, our analysis implies the identifications

$$
\begin{aligned}
& B_{\mu m_{1} \ldots m_{5}}(x, y)=-\frac{1}{4 \cdot 6 !}\left(K_{m_{1} \ldots m_{5}}^{I J}-6 \cdot 6 ! \stackrel{\circ}{\zeta}_{m_{1} \ldots m_{5} p} K^{p I J}\right)(y) A_{\mu}^{I J}(x),
\end{aligned}
$$

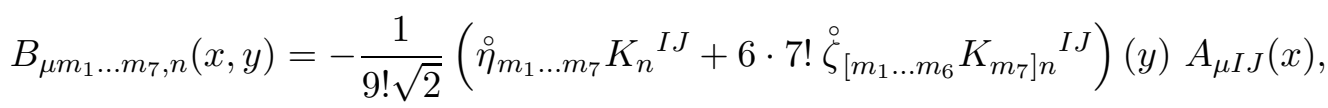

where

$$
K_{m_{1} \ldots m_{5}}^{I J}=i \stackrel{\circ}{e}_{m_{1}}^{a_{1}} \ldots \stackrel{\circ}{e}_{m_{5}}^{a_{5}} \bar{\eta}^{I} \Gamma_{a_{1} \ldots a_{5}} \eta^{J}
$$

\footnotetext{
${ }^{6}$ Note that the difference between the coefficient in the ansatz for $B_{\mu m n}$ here and in Ref. [2] is due to differing conventions for $F=$ A. In [2], this constant was fixed based on the tests of the non-linear flux ansatz in 3] where $F=4 \partial A$. However, here, as in much of the related literature, we use the convention $F=4 ! \partial A$.
} 
is again a bilinear in the Killing spinors. The quantity $\stackrel{\circ}{\zeta}_{m_{1} \ldots m_{6}}(y)$ is defined such that

$$
7 ! \partial_{\left[m_{1}\right.} \stackrel{\circ}{\zeta}_{\left.m_{2} \ldots m_{7}\right]}=m_{7} \stackrel{\circ}{\eta}_{m_{1} \ldots m_{7}},
$$

and is thus to be regarded as a potential for the volume form on the round seven-sphere.

While the first terms on the right hand side of the ansätze (45) and (46) are not completely unexpected, the crucial new feature in comparison with formulae (39) and (40) is the presence of the non-globally defined field $\stackrel{\circ}{\zeta}_{m_{1} \ldots m_{6}}$. One way of understanding its presence in the above ansätze is to observe that the components of the field strength $F_{(4)}$ along the four dimensions $F_{\mu \nu \rho \sigma}$ is non-zero and, for maximally symmetric solutions is proportional to the volume form in four dimensions [6]. Equivalently, this non-zero component of the three-form potential $A_{\mu \nu \rho}$ can be viewed as a non-zero component of its six-form dual along the seven-dimensional directions [4, namely

$$
A_{m_{1} \ldots m_{6}} \sim \stackrel{\circ}{\zeta}_{m_{1} \ldots m_{6}}
$$

Thus, even for the $S^{7}$ solution [7] where the scalar expectation values are essentially trivial, one would have to obtain such a non-zero value for $A_{m_{1} \ldots m_{6}}$ from a non-linear ansatz. Indeed, the coefficients in ansätze (45) and (46) have been fixed by requiring consistency with the $S^{7}$ solution. In fact, the 'vacuum expectation value' of the six-form field will be non-vanishing for any non-trivial compactification (that is, other than the $T^{7}$ reduction of [14]) of $D=11$ supergravity.

As before, comparing the four-dimensional supersymmetry transformations (37) and (38) with their higher-dimensional analogues (9) and (10) gives

$$
\begin{aligned}
& e_{m_{1} \ldots m_{5} i j}(x, y)=\frac{1}{6 ! \sqrt{2}}\left(K_{m_{1} \ldots m_{5}}^{I J}-6 \cdot 6 ! \stackrel{\circ}{\zeta}_{m_{1} \ldots m_{5} p} K^{p I J}\right)(y)\left[u_{i j}{ }^{I J}+v_{i j I J}\right](x), \\
& e_{m_{1} \ldots m_{7}, n i j}(x, y)=\frac{2}{9 !} i\left(\stackrel{\circ}{\eta}_{m_{1} \ldots m_{7}} K_{n}^{I J}+6 \cdot 7 ! \stackrel{\circ}{\zeta}_{\left[m_{1} \ldots m_{6}\right.} K_{\left.m_{7}\right] n}^{I J}\right)(y)\left[u_{i j}{ }^{I J}-v_{i j I J}\right](x),
\end{aligned}
$$

where we have again converted 'curved' to 'flat' $\mathrm{SU}(8)$ indices by means of relations (41).

The fact that we now have two expressions for the generalised vielbeine, one in terms of elevendimensional fields, equations (11)-(14), and one in terms of four-dimensional scalars, equations (43), (44), (50) and (51), allows us to derive non-linear ansätze for internal fields. The non-linear ansatz for the metric [26] is found by considering the expression

$$
e^{m}{ }_{A B} e^{n A B},
$$

which gives [26]

$$
\Delta^{-1} g^{m n}(x, y)=\frac{1}{8} K^{m I J} K^{n K L}(y)\left[\left(u_{I J}^{i j}+v^{i j I J}\right)\left(u_{i j}{ }^{K L}+v_{i j K L}\right)\right](x) .
$$

Similarly, the non-linear flux ansatz [2] is found by considering the expression

$$
e_{m n}^{A B} e^{p}{ }_{A B}
$$

which gives 7

$$
A_{m n p}(x, y)=-\frac{\sqrt{2}}{96} i \Delta g_{p q}(x, y) K_{m n}{ }^{I J} K^{q K L}(y)\left[\left(u^{i j}{ }_{I J}-v^{i j I J}\right)\left(u_{i j}{ }^{K L}+v_{i j K L}\right)\right](x),
$$

\footnotetext{
${ }^{7}$ See footnote 6 for an explanation of the extra factor of 6 between this expression and the non-linear flux ansatz in [2, 3].
} 
where one uses the metric ansatz (53) to compute $\Delta g_{p q}$ in the equation above. Now, considering

$$
e_{m_{1} \ldots m_{5} A B} e^{n A B}
$$

gives a non-linear ansatz for the internal six-form components. It is simple to show that by equating the contraction of the two vielbeine using definitions (11) and (13) on one side and definitions (43) and (50) on the other gives

$$
\begin{aligned}
& A_{n m_{1} \ldots m_{5}}-\frac{\sqrt{2}}{4} A_{n\left[m_{1} m_{2}\right.} A_{\left.m_{3} m_{4} m_{5}\right]} \\
& \quad=-\frac{\sqrt{2}}{16 \cdot 6 !} \Delta g_{n p}\left(K_{m_{1} \ldots m_{5}}^{I J}-6 \cdot 6 ! \stackrel{\circ}{\zeta}_{m_{1} \ldots m_{5} q} K^{q I J}\right) K^{p K L}\left[\left(u_{i j}{ }^{I J}+v_{i j I J}\right)\left(u^{i j} K L+v^{i j K L}\right)\right],
\end{aligned}
$$

where the internal metric and three-form potential components are derived using ansätze (53) and (55). We note that the complete antisymmetry of $A_{m_{1} \ldots m_{6}}$ in all six indices is not manifest from this expression; our explicit tests for the $\mathrm{SO}(7)^{ \pm}$invariant solutions (see section 5) show, however, that this consistency requirement is met in non-trivial examples.

An ansatz for the six-form potential can also be obtained by considering the fourth generalised vielbeine $e_{m_{1} \ldots m_{7}, n A B}$. The relation of this ansatz to the ansatz (57) above will not be obvious, but clearly the two ansätze must be equivalent. This completes the set of uplift ansätze for all bosonic degrees of freedom from maximal gauged supergravity to eleven dimensions.

\section{Generalised vielbein postulates and the $S^{7}$ compactification}

The generalised vielbein postulate for $e_{A B}^{m}$ plays an important role in establishing the consistency of the $S^{7}$ reduction of $D=11$ supergravity [32, 33]. In particular, in [32] it is shown that upon the $S^{7}$ compactification, the $d=4$ generalised vielbein postulate reduces to the $\mathrm{E}_{7(7)}$ Cartan equation of gauged maximal supergravity, to wit

$$
\mathcal{V}^{-1}(x)\left(\partial_{\mu}-g A_{\mu}^{I J}(x) X^{I J}\right) \mathcal{V}(x)=Q_{\mu}(x)+P_{\mu}(x),
$$

where $X^{I J}$ generate the compact $\mathrm{SO}(8)$ subgroup inside $\mathrm{SL}(8, \mathbb{R}) \subset \mathrm{E}_{7(7)}$, and $g$ is the gauge coupling constant. In this section, then, we explore the generalised vielbein postulates in a more general context than [32, 33] by investigating the full set of relations (24)-(27) for the extra vielbein components (11)-(14), hence taking into account the full set of 56 vector fields identified in section 2.1. The presence of both electric and magnetic vectors in these relations indicates that our construction should eventually allow one to derive more general gaugings of $N=8$ supergravity from compactification, and thereby to understand how the embedding tensor emerges from the $D=11$ theory upon different compactifications. However, here we will concentrate on the $S^{7}$ compactification, as this case already by itself provides a wealth of new insights, in particular concerning the role of dual vector fields in non-abelian gaugings. We will briefly return to the more general case in the final section, postponing a detailed discussion to later work.

One issue that we will specifically address and resolve in this section is the following: The fact that not all of the 56 vector fields can correspond to independent propagating degrees of freedom, and the generic emergence of non-abelian gauge interactions for non-trivial compactifications (for which the standard abelian dualisation linking electric and magnetic vectors no longer works), immediately

raises the question of how the theory can dispose of the unwanted vectors and thereby ensure the 
consistency of compactified theory. Here we will establish consistency of the equations for the $S^{7}$ compactification by explicitly showing how the magnetic vector fields drop out of the generalised vielbein postulates, leaving only electric gaugings.

For the $S^{7}$ compactification the reduction ansatz of the vector fields and the generalised vielbeine are given by equations (39), (40), (45), (46), and equations (43), (44), (50), (51), respectively. To adapt to the $S^{7}$ compactification we introduce a minor modification by replacing (28) by

$$
\mathcal{D}_{\mu}=\partial_{\mu}-B_{\mu}^{m} \stackrel{\circ}{D}_{m}
$$

and the connections (29) and (30) by

$$
\begin{gathered}
\mathcal{Q}_{\mu B}^{A}=-\frac{1}{2}\left[e^{m}{ }_{a} \stackrel{\circ}{D}_{m} B_{\mu}{ }^{n} e_{n b}-\left(e^{p}{ }_{a} \mathcal{D}_{\mu} e_{p b}\right)\right] \Gamma_{A B}^{a b}-\frac{\sqrt{2}}{12} \Delta^{-1 / 2} e_{\mu}^{\prime \alpha}\left(F_{\alpha a b c} \Gamma_{A B}^{a b c}-\eta_{\alpha \beta \gamma \delta} F^{\beta \gamma \delta a} \Gamma_{a A B}\right), \\
\mathcal{P}_{\mu A B C D}=\frac{3}{4}\left[e^{m}{ }_{a} \stackrel{\circ}{D}_{m} B_{\mu}{ }^{n} e_{n b}-\left(e^{p}{ }_{a} \mathcal{D}_{\mu} e_{p b}\right)\right] \Gamma_{[A B}^{a} \Gamma_{C D]}^{b}-\frac{\sqrt{2}}{8} \Delta^{-1 / 2} e_{\mu}^{\prime \alpha} F_{a b c \alpha} \Gamma_{[A B}^{a} \Gamma_{C D]}^{b c} \\
-\frac{\sqrt{2}}{48} \Delta^{-1 / 2} e_{\mu \alpha}^{\prime} \eta^{\alpha \beta \gamma \delta} F_{a \beta \gamma \delta} \Gamma_{b[A B} \Gamma_{C D]}^{a b},
\end{gathered}
$$

that is, replacing the partial derivative $\partial_{m}$ by the $S^{7}$ background covariant derivative $\stackrel{\circ}{D}_{m}$ everywhere. Likewise this replacement is to be made everywhere in the vielbein postulate equations (24)-(27).

As before (cf. (41)) we have to convert SU(8) indices in order to relate the connection coefficients above to their four-dimensional counterparts for the $S^{7}$ compactification. This change of basis is covariant for all fields, with the exception of 32 ]

$$
\mathcal{Q}_{\mu j}^{i}=\eta_{A}^{i} \eta_{j}^{B}\left(\mathcal{Q}_{\mu B}^{A}-i \frac{\sqrt{2}}{4} m_{7} A_{\mu}{ }^{K L} K^{n K L} \stackrel{\circ}{e}_{n}{ }^{a} \Gamma_{a A B}\right)
$$

Using the Killing spinor equation and the equation above,

$$
\begin{aligned}
\mathcal{D}_{\mu} e_{A B}^{m}+\mathcal{Q}_{\mu[A}^{C} e_{B] C}^{m} & =\left(\partial_{\mu}-B_{\mu}{ }^{m} \stackrel{\circ}{D}_{m}\right)\left[\eta_{A}^{i}(y) \eta_{B}^{j}(y) e_{i j}^{m}(x, y)\right]+\mathcal{Q}_{\mu}^{C}\left[A e_{B] C}^{m}\right. \\
& =\eta_{A}^{i} \eta_{B}^{j} \mathcal{D}_{\mu} e_{i j}^{m}+i m_{7} B_{\mu}{ }^{m} \stackrel{\circ}{e}_{m}{ }^{a} \Gamma^{a}{ }_{C[A} e_{B] C}^{m}+\mathcal{Q}_{\mu}^{C}\left[A e_{B] C}^{m}\right. \\
& =\eta_{A}^{i} \eta_{B}^{j}\left(\mathcal{D}_{\mu} e_{i j}^{m}+\mathcal{Q}_{\mu[i}^{k} e_{j] k}^{m}\right) .
\end{aligned}
$$

Analogous relations hold for the other generalised vielbeine:

$$
\begin{aligned}
& \mathcal{D}_{\mu} e_{m n A B}+\mathcal{Q}_{\mu[A}^{C} e_{m n B] C}=\eta_{A}^{i} \eta_{B}^{j}\left(\mathcal{D}_{\mu} e_{m n i j}+\mathcal{Q}_{\mu[i}^{k} e_{m n j] k}\right), \\
& \mathcal{D}_{\mu} e_{m_{1} \ldots m_{5} A B}+\mathcal{Q}_{\mu[A}^{C} e_{\left.m_{1} \ldots m_{5} B\right] C}=\eta_{A}^{i} \eta_{B}^{j}\left(\mathcal{D}_{\mu} e_{m_{1} \ldots m_{5} i j}+\mathcal{Q}_{\mu[i}^{k} e_{\left.m_{1} \ldots m_{5} j\right] k}\right), \\
& \mathcal{D}_{\mu} e_{m_{1} \ldots m_{7}, n A B}+\mathcal{Q}_{\mu[A}^{C} e_{\left.m_{1} \ldots m_{7}, n B\right] C}=\eta_{A}^{i} \eta_{B}^{j}\left(\mathcal{D}_{\mu} e_{m_{1} \ldots m_{7}, n i j}+\mathcal{Q}_{\mu[i}^{k} e_{\left.m_{1} \ldots m_{7}, n j\right] k}\right) \text {. }
\end{aligned}
$$

The non-covariant term in (62) thus ensures that we can freely convert between 'curved' and 'flat' $\mathrm{SU}(8)$ indices in all relations.

Let us first consider the generalised vielbein postulate for $e_{A B}^{m}$, which is already analysed in [32]. The supersymmetry transformation of the graviphoton $B_{\mu}{ }^{m}$ gives rise to the generalised vielbein $e_{A B}^{m}$, equation (77). In Kaluza-Klein theory, the exact ansatz relating the graviphoton to the fourdimensional vector field is given by the the Killing vectors of the internal space, (39). As we have already mentioned, this ansatz, via equations (37) and (77), also furnishes an ansatz (43) for 
the generalised vielbein $e_{A B}^{m}$. The emergence of the $\mathrm{SO}(8)$ covariantisation of the four-dimensional derivative is then easily seen to be a consequence, in accordance with general Kaluza-Klein theory, of the appearance of the commutator of two Killing vector fields in

$$
\left(\partial_{\mu}-B_{\mu}^{n} \stackrel{\circ}{D}_{n}\right) e_{i j}^{m}+\stackrel{\circ}{D}_{n} B_{\mu}^{m} e_{i j}^{n}
$$

and the fact that $\stackrel{\circ}{D}_{m} B_{\mu}{ }^{m}=0$ for any Killing vector. More precisely, plugging these ansätze into the generalised vielbein postulate (24) and using equation (63), the latter reduces to

$$
\begin{gathered}
\partial_{\mu} e_{i j}^{m}-\frac{\sqrt{2}}{8} \stackrel{\circ}{D}_{n} K^{n I J} A_{\mu}{ }^{I J} e_{i j}^{m}+\frac{\sqrt{2}}{4}\left(K^{n I J} \stackrel{\circ}{D}_{n} K^{m K L}-K^{n K L} \stackrel{\circ}{D}_{n} K^{m I J}\right) A_{\mu}{ }^{I J}\left[u_{i j}{ }^{K L}+v_{i j K L}\right] \\
+\mathcal{Q}_{\mu[i}^{k} e_{j] k}^{m}+\mathcal{P}_{\mu i j k l} e^{m k l}=0 .
\end{gathered}
$$

Using equation (36), the first three terms in the generalised vielbein postulate (68) reduce to

$$
K^{m I J}\left(\delta_{K}^{I} \partial_{\mu}-2 \sqrt{2} A_{\mu}{ }^{I K}\right)\left[u_{i j}{ }^{K J}+v_{i j K J}\right]=0,
$$

which is a contraction of the $\mathrm{SO}(8)$ gauge covariant derivative on the scalar fields. Denoting

$$
w^{+}{ }_{i j}^{I J}=u_{i j}^{I J}+v_{i j I J}, \quad w^{-}{ }_{i j}^{I J}=i\left[u_{i j}^{I J}-v_{i j I J}\right]
$$

and by $w^{+i j}$ IJ and $w^{-i j}$ IJ their complex conjugates, respectively, the generalised vielbein postulate gives

$$
K^{m I J}\left(D_{\mu}^{\mathrm{SO}(8)} w^{+}{ }_{i j}{ }^{I J}+\mathcal{Q}_{\mu[i}^{k} w^{+}{ }_{j] k}{ }^{I J}+\mathcal{P}_{\mu i j k l} w^{+k l}{ }_{I J}\right)=0,
$$

where the $\mathrm{SO}(8)$ gauge covariant derivative is defined as

$$
\left.D_{\mu}^{\mathrm{SO}(8)} w^{ \pm}{ }_{i j}{ }^{I J}=\partial_{\mu} w^{ \pm}{ }_{i j}{ }^{I J}-2 \sqrt{2} m_{7} A_{\mu}{ }^{K[I} w^{ \pm}{ }_{i j} J\right] K .
$$

Thence, we identify $A_{\mu}{ }^{I J}$ as the $\mathrm{SO}(8)$ gauge fields and $\sqrt{2} m_{7}$ as the $\mathrm{SO}(8)$ gauge coupling. Thus, the generalised vielbein postulate reduces to a particular component of the $\mathrm{E}_{7(7)}$ Cartan equation with $\mathrm{SO}(8)$ covariant derivatives, as claimed above.

While this part of the argument was already given in [32, the $\mathrm{SO}(8)$ covariantisation on the other components of the generalised vielbein cannot be traced back to geometrical arguments of this type. In Ref. [32] it is argued that equation (71) in fact implies the $\mathrm{E}_{7(7)}$ Cartan equation with $\mathrm{SO}(8)$ covariant derivatives. However, here, by considering all of the generalised vielbein postulates, we can see that this equation follows directly upon compactification on $S^{7}$. In other words, the rest of the generalised vielbein postulates give rise to the 'missing' components of the Cartan equation in (58). We will show this in turn for each of the generalised vielbein postulates (25) -(27).

The generalised vielbein postulate for $e_{m n A B}$, (25), becomes, after conversion to 'flat' $\mathrm{SU}(8)$ indices using equation (64) and again using $\stackrel{\circ}{D}_{m} B_{\mu}{ }^{m}=0$,

$$
\mathcal{D}_{\mu} e_{m n i j}+2 \stackrel{\circ}{D}_{[m} B_{|\mu|} e_{n] p i j}+3 \stackrel{\circ}{D}_{[m} B_{|\mu| n p]} e_{i j}^{p}+\mathcal{Q}_{\mu[i}^{k} e_{m n j] k}+\mathcal{P}_{\mu i j k l} e_{m n}{ }^{k l}=0 .
$$

The new feature here is the presence of the 'magnetic' vectors $B_{\mu m n}$, which according to equation (40) could in principle lead to gauging of magnetic vector fields in the four-dimensional theory. However, note that the relations between $e_{m n A B}$, (44), and $B_{\mu m n}$, (40), and the four-dimensional fields are not made with respect to Killing vectors but via the tensor $K_{m n}^{I J}$, which from the Killing spinor equation satisfies

$$
\stackrel{\circ}{D}_{p} K_{m n}^{I J}=2 m_{7} \stackrel{\circ}{g}_{p[m} K_{n]}^{I J}
$$


This immediately implies that

$$
\stackrel{\circ}{D}_{[m} B_{|\mu| n p]}=0 .
$$

Hence, the magnetic vector fields drop out of relation (25) in the $S^{7}$ reduction, thus ensuring that effectively only the 28 electric vectors appear in the four-dimensional theory with their non-abelian interactions, while the magnetic vectors all decouple! Using equations (34) and (74), the generalised vielbein postulate then further simplifies to

$$
\partial_{\mu} e_{m n i j}+\frac{1}{12} m_{7} A_{\mu}{ }^{I J} w^{-}{ }_{i j}{ }^{K L}\left(K^{p}{ }_{[m}^{I J} K_{n] p}{ }^{K L}-K_{[m}{ }^{I J} K_{n]}{ }^{K L}\right)+\mathcal{Q}_{\mu[i}^{k} e_{m n j] k}+\mathcal{P}_{\mu i j k l} e_{m n}{ }^{k l}=0,
$$

which, using equation (136), gives another component of the $\mathrm{E}_{7(7)}$ Cartan equation:

$$
K_{m n}{ }^{I J}\left(D_{\mu}^{\mathrm{SO}(8)} w^{-}{ }_{i j}^{I J}+\mathcal{Q}_{\mu[i}^{k} w^{-}{ }_{j] k}^{I J}+\mathcal{P}_{\mu i j k l} w^{-k l}{ }_{I J}\right)=0 .
$$

Next we consider the third equation, (26), which becomes, using $\stackrel{\circ}{D}_{m} B_{\mu}{ }^{m}=0$ and equations (65) and (75),

$$
\begin{aligned}
\partial_{\mu} e_{m_{1} \ldots m_{5} i j}-B_{\mu}{ }^{p} \stackrel{\circ}{D}_{p} e_{m_{1} \ldots m_{5} i j}-5 \stackrel{\circ}{D}_{\left[m_{1}\right.} B_{|\mu|}{ }^{p} e_{\left.m_{2} \ldots m_{5}\right] p i j}-6 \stackrel{\circ}{D}_{\left[m_{1}\right.} B_{\left.|\mu| m_{2} \ldots m_{5} p\right]} e_{i j}^{p} \\
+\mathcal{Q}_{\mu[i}^{k} e_{\left.m_{1} \ldots m_{5} j\right] k}+\mathcal{P}_{\mu i j k l} e_{m_{1} \ldots m_{5}}{ }^{k l}=0 .
\end{aligned}
$$

In this case the reduction ansätze (45) and (50) not only contain tensors, rather than Killing vectors, but they also contain the potential for the volume-form on the round 7 -sphere $\stackrel{\circ}{\zeta}$, which is not globally defined. As we shall see below, these terms are not only crucial for obtaining the correct non-linear flux ansätze, but equally crucial in the reduction of the generalised vielbein postulate.

Inserting the reduction ansätze for the generalised vielbeine and the vector fields and using equations (133), (34) and (74), we obtain

$$
\begin{aligned}
& B_{\mu}{ }^{p} \stackrel{\circ}{D}_{p} e_{m_{1} \ldots m_{5} i j}=\frac{1}{4 \cdot 6 !} A_{\mu}{ }^{I J} w^{+}{ }_{i j}{ }^{K L} K^{p I J}\left(m_{7} \stackrel{\circ}{\eta}_{m_{1} \ldots m_{5} p q} K^{q K L}+6 \cdot 6 ! \stackrel{\circ}{D}_{p} \stackrel{\circ}{\zeta}_{m_{1} \ldots m_{5} q} K^{q K L}\right. \\
& \left.+6 \cdot 6 ! m_{7} \stackrel{\circ}{\zeta}_{m_{1} \ldots m_{5} q} K_{p}^{q}{ }^{K L}\right) \\
& \stackrel{\circ}{D}_{\left[m_{1}\right.} B_{\left.|\mu| m_{2} \ldots m_{5} p\right]} e_{i j}^{p}=-\frac{1}{4 \cdot 6 !} A_{\mu}^{I J} w^{+}{ }_{i j} K L K^{p K L}\left(m_{7}{\stackrel{\circ}{\eta \ldots m_{5} p q}}^{q I J}-6 \cdot 6 ! \stackrel{\circ}{D}_{\left[m_{1}\right.} \stackrel{\circ}{\zeta}_{\left.m_{2} \ldots m_{5} p\right] q} K^{q I J}\right. \\
& \left.-6 \cdot 6 ! m_{7} \stackrel{\circ}{\zeta}_{q\left[m_{1} \ldots m_{5}\right.} K_{p]}^{q}{ }^{I J}\right) \text {. }
\end{aligned}
$$

Hence,

$$
\begin{aligned}
& B_{\mu}^{p} \stackrel{\circ}{D}_{p} e_{m_{1} \ldots m_{5} i j}+6 \stackrel{\circ}{D}_{\left[m_{1}\right.} B_{\left.|\mu| m_{2} \ldots m_{5} p\right]} e_{i j}^{p} \\
& =\frac{1}{4 \cdot 6 !} A_{\mu}^{I J} w^{+}{ }_{i j}^{K L}\left(7 m_{7} \stackrel{\circ}{\eta}_{m_{1} \ldots m_{5} p q} K^{p I J} K^{q K L}+6 \cdot 7 ! \stackrel{\circ}{D}_{[p} \stackrel{\circ}{\zeta}_{\left.m_{1} \ldots m_{5} q\right]} K^{p I J} K^{q K L}\right. \\
& \left.+6 \cdot 6 ! m_{7} \stackrel{\circ}{\zeta}_{m_{1} \ldots m_{5} q} K^{p I J} K_{p}^{q}{ }^{K L}+36 \cdot 6 ! m_{7} \stackrel{\circ}{\zeta}_{q\left[m_{1} \ldots m_{5}\right.} K_{p]}^{q}{ }_{p]}^{I J} K^{p K L}\right), \\
& =\frac{1}{4 \cdot 6 !} m_{7} A_{\mu}^{I J} w^{+}{ }_{i j} K L\left({\stackrel{\circ}{\eta_{m} \ldots m_{5} p q}} K^{p I J} K^{q K L}+48 \cdot 6 ! \stackrel{\circ}{\zeta}_{m_{1} \ldots m_{5} q} \delta^{I K} K^{q J L}\right. \\
& \left.\left.+30 \cdot 6 ! \stackrel{\circ}{\zeta}_{p q\left[m_{1} \ldots m_{4}\right.} K^{q}{ }_{m_{5}}\right] K^{I J} K^{p K L}\right),
\end{aligned}
$$


where in the second equality above we have used equations (48) and (135). A straightforward substitution of the ansätze (39) and (50) also gives

$$
5 \stackrel{\circ}{D}_{\left[m_{1}\right.} B_{|\mu|}{ }^{p} e_{\left.m_{2} \ldots m_{5}\right] p i j}=\frac{5 m_{7}}{8 \cdot 6 !} A_{\mu}{ }^{I J} w^{+}{ }_{i j}{ }^{K L} K_{\left[m_{1}\right.}{ }^{I J}\left(\stackrel{\circ}{\eta}_{\left.m_{2} \ldots m_{5}\right] p q r} K^{q r K L}+12 \cdot 6 ! \stackrel{\circ}{\zeta}_{\left.m_{2} \ldots m_{5}\right] p q} K^{q K L}\right) .
$$

Now, using the identity

$$
5 K_{\left[m_{1}\right.}^{{ }^{I J}}{\stackrel{\circ}{\eta_{\left.m_{2} \ldots m_{5}\right] p q r}}}^{q r K L}=2 K_{r}^{p}{ }_{r}^{I J}{\stackrel{\circ}{m_{1} \ldots m_{5} p q}} K^{q r K L}
$$

and equation (136), the first terms on the right hand side of equations (81) and (82) precisely combine to give

$$
\frac{1}{6 !} m_{7} A_{\mu}^{I J} w^{+}{ }_{i j}{ }^{K L}{\stackrel{\circ}{\eta_{m_{1} \ldots m_{5} p q}}}^{I K} K^{p q J L}=-\frac{2}{6 !} m_{7} A_{\mu}^{I J} w^{+}{ }_{i j}^{K L} \delta^{I K} K_{m_{1} \ldots m_{5}}{ }^{J L} .
$$

Moreover, the third term on the right hand side of equation (81) cancels the second term on the right hand side of equation (82). Therefore, in all, equation (78) simplifies to

$$
\left(K_{m_{1} \ldots m_{5}}{ }^{I J}-6 \cdot 6 ! \stackrel{\circ}{\zeta}_{m_{1} \ldots m_{5} p} K^{p I J}\right)\left[D_{\mu}^{\mathrm{SO}(8)} w^{+}{ }_{i j}{ }^{I J}+\mathcal{Q}_{\mu[i}^{k} w^{+}{ }_{j] k}{ }^{I J}+\mathcal{P}_{\mu i j k l} w^{+k l}{ }_{I J}\right]=0 .
$$

Using equations (71) and (133), the above equation implies

$$
K^{m n I J}\left(D_{\mu}^{\mathrm{SO}(8)} w^{+}{ }_{i j}{ }^{I J}+\mathcal{Q}_{\mu[i}^{k} w^{+}{ }_{j] k}{ }^{I J}+\mathcal{P}_{\mu i j k l} w^{+k l}{ }_{I J}\right)=0 .
$$

Finally, we consider the generalised vielbein postulate for $e_{m_{1} \ldots m_{7}, n}$, which using the same equations as before, simplifies to

$$
\begin{aligned}
\partial_{\mu} e_{m_{1} \ldots m_{7}, n i j}-B_{\mu}{ }^{p} \stackrel{\circ}{D}_{p} e_{m_{1} \ldots m_{7}, n i j}-\partial_{n} B_{\mu}{ }^{p} e_{m_{1} \ldots m_{7}, p i j}-2 \partial_{\left[m_{1}\right.} B_{|\mu| m_{2} \ldots m_{6}} e_{\left.m_{7}\right] n i j} & \\
& +\mathcal{Q}_{\mu[i}^{k} e_{\left.m_{1} \ldots m_{7}, n j\right] k}+\mathcal{P}_{\mu i j k l} e_{m_{1} \ldots m_{7}, n}{ }^{k l}=0 .
\end{aligned}
$$

A similar calculation to the one outlined above for the $e_{m_{1} \ldots m_{5}} A B$ gives

$$
\begin{aligned}
& B_{\mu}{ }^{p} \stackrel{\circ}{D}_{p} e_{m_{1} \ldots m_{7}, n i j}=-\frac{\sqrt{2}}{2 \cdot 9 !} A_{\mu}{ }^{I J} w^{-}{ }_{i j} K L\left(5 m_{7}{\stackrel{\circ}{\eta_{m} \ldots m_{7}}} K^{p I J} K_{p n}^{K L}+36 \cdot 7 ! \stackrel{\circ}{D}_{\left[m_{1} \mid\right.} \stackrel{\circ}{\zeta}_{p \mid m_{2} \ldots m_{6}} K^{p I J} K_{\left.m_{7}\right] n}^{K L}\right. \\
& \left.+6 \cdot 7 ! m_{7} \stackrel{\circ}{\zeta}_{\left[m_{1} \ldots m_{6}\right.}\left(K_{\left.m_{7}\right]}^{I J} K_{n}^{K L}-K_{\left.m_{7}\right]}^{K L} K_{n}^{I J}\right)\right),
\end{aligned}
$$

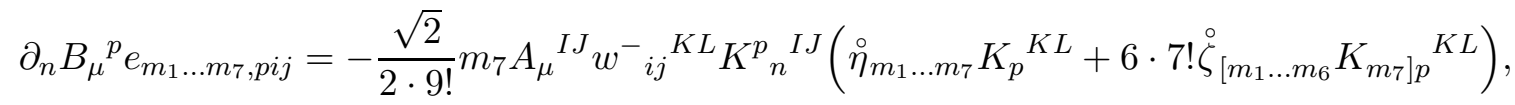

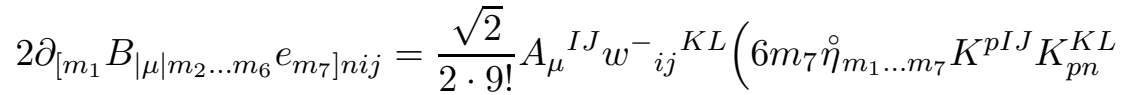

$$
\begin{aligned}
& +36 \cdot 7 ! \stackrel{\circ}{D}_{\left[m_{1} \mid\right.} \stackrel{\circ}{\zeta}_{p \mid m_{2} \ldots m_{6}} K^{p I J} K_{\left.m_{7}\right] n}^{K L} \\
& \left.+6 \cdot 7 ! m_{7} \stackrel{\circ}{\zeta}_{\left[m_{1} \ldots m_{6}\right.} K_{\left.m_{7}\right] p}{ }^{I J} K_{n}^{p}{ }_{n}^{K L}\right),
\end{aligned}
$$


where we have used equations (34), (48), (174), (133) and antisymmetrisations over eight indices, which vanish, to simplify the expressions above. It is now simple to verify, using equations (135) and (136), that

$$
\begin{aligned}
& B_{\mu}{ }^{p} \stackrel{\circ}{D}_{p} e_{m_{1} \ldots m_{7}, n i j}+\partial_{n} B_{\mu}{ }^{p} e_{m_{1} \ldots m_{7}, p i j}+2 \partial_{\left[m_{1}\right.} B_{|\mu| m_{2} \ldots m_{6}} e_{\left.m_{7}\right] n i j} \\
& =\frac{4 \sqrt{2}}{9 !} m_{7} A_{\mu}{ }^{K I} w^{-}{ }_{i j} J K\left(\stackrel{\circ}{\eta}_{m_{1} \ldots m_{7}} K_{n}^{I J}+6 \cdot 7 ! \stackrel{\circ}{\zeta}_{\left[m_{1} \ldots m_{6}\right.} K_{\left.m_{7}\right] n}{ }^{I J}\right) .
\end{aligned}
$$

Hence equation (87) reduces to

$$
K_{m}^{I J}\left(D_{\mu}^{\mathrm{SO}(8)} w^{-}{ }_{i j}^{I J}+\mathcal{Q}_{\mu[i}^{k} w^{-}{ }_{j] k}^{I J}+\mathcal{P}_{\mu i j k l} w^{-k l}{ }_{I J}\right)=0,
$$

where we have used equation (177) to eliminate the expression proportional to $\stackrel{\circ}{\zeta}$.

To sum up: in the reduction of (24), the $\mathrm{SO}(8)$ gauge covariant derivative arose from the geometrical properties of Killing vectors on $S^{7}$. However, for the other generalised vielbeine the emergence of the $\mathrm{SO}(8)$ gauge covariant derivative is not so direct. Indeed, the reduction of the other generalised vielbein postulates is particularly novel given that the postulates (25)-(27) contain fields $\left(B_{\mu m n}\right.$ and $\left.B_{\mu m_{1} \ldots m_{5}}\right)$ for which the identification with the four-dimensional vector fields is not made with the $S^{7}$ Killing vectors, but with more general structures on the 7 -sphere. We stress again that in the derivation of the last two equations, (86) and (92), the $\stackrel{\circ}{\zeta}$ terms in the ansätze are crucial for obtaining the $\mathrm{SO}(8)$ gauge covariant terms. Therefore, the $\mathrm{SO}(8)$ gauge covariant derivatives emerge, not in spite of but because of these more general structures.

The results that we have obtained from the reduction of the generalised vielbein postulates, equations (71), (77), (86) and (92), can be summarised as

$$
\begin{aligned}
& K^{a I J}\left(D_{\mu}^{\mathrm{SO}(8)} w^{ \pm}{ }_{i j}{ }^{I J}+\mathcal{Q}_{\mu[i}^{k} w^{ \pm}{ }_{j] k}{ }^{I J}+\mathcal{P}_{\mu i j k l} w^{ \pm k l}{ }_{I J}\right)=0, \\
& K^{a b I J}\left(D_{\mu}^{\mathrm{SO}(8)} w^{ \pm}{ }_{i j}{ }^{I J}+\mathcal{Q}_{\mu[i}^{k} w^{ \pm}{ }_{j] k}{ }^{I J}+\mathcal{P}_{\mu i j k l} w^{ \pm k l}{ }_{I J}\right)=0 .
\end{aligned}
$$

Since $K^{a I J}$ and $K^{a b I J}$ form a basis of antisymmetric $28 \times 28$ matrices, these equations are equivalent to

$$
D_{\mu}^{\mathrm{SO}(8)} \mathcal{V}_{i j}^{I J}+\mathcal{Q}_{\mu[i}^{k} \mathcal{V}_{j] k}^{I J}+\mathcal{P}_{\mu i j k l} \mathcal{V}^{k l}{ }_{I J}=0,
$$

where $\mathcal{V}(x)$ is the $\mathrm{E}_{7(7)} / \mathrm{SU}(8)$ coset element parametrised by the scalar fields. In Ref. [32 this equation was argued, somewhat indirectly, to hold solely on the basis of the generalised vielbein postulate for $e_{A B}^{m}$, equation (24). Here we see that it naturally follows from the full set of generalised vielbein postulates.

In summary, we find that, in the case of the $S^{7}$ compactification both $B_{\mu}^{m}$ and $B_{\mu m_{1} \ldots m_{5}}$ contribute to the electric vector fields, while the magnetic vector fields drop out of the expressions. Indeed, from equations (39) and (45) we see that this is natural because $B_{\mu}{ }^{m}$ and $B_{\mu m_{1} \ldots m_{5}}$ project onto different $\mathrm{SO}(8)$ components of the electric vector field $A_{\mu}{ }^{I J}$.

\section{A first test of the non-linear six-form ansatz}

In this section we check the consistency of the relations derived in section 3 , in particular the non-linear ansatz for the dual six-form using the relatively simple, yet non-trivial $\mathrm{SO}(7)^{ \pm}$invariant solutions of gauged supergravity [29] for which the higher-dimensional solutions are known [27, 28]. 
For the convenience of the reader, we give a brief description of these solutions from both a four and higher-dimensional perspective in appendix $\mathrm{A}$. The non-linear metric and flux ansätze, equations (53) and (55), respectively, have been subjected to some very non-trivial tests, which they have passed with remarkable success, most recently in [3] (where references to earlier work can also be found). In particular, these ansätze correctly reproduce the $\mathrm{SO}(7)^{ \pm}$invariant solutions [26, 33, 3]. Therefore, let us consider the non-linear ansatz, equation (57), for the dual six-form potential of the $\mathrm{SO}(7)^{ \pm}$invariant solutions.

Using the following $\mathrm{E}_{7(7)}$ properties satisfied by $u^{i j}{ }_{K L}$ and $v^{i j K L}$ [8]

$$
\begin{aligned}
& u_{I J}^{i j} u_{i j}{ }^{K L}-v_{i j I J} v^{i j K L}=\delta_{I J}^{K L}, \\
& u_{I J}^{i j} v_{i j K L}-v_{i j I J} u^{i j}{ }_{K L}=0,
\end{aligned}
$$

one can show that

$$
\left(u_{i j}^{I J}+v_{i j I J}\right)\left(u_{K L}^{i j}+v^{i j K L}\right)=\delta_{K L}^{I J}+2 v_{I J M N} v^{K L M N}+2 \operatorname{Re}\left(u_{M N}^{I J} v^{M N K L}\right) .
$$

Now, using the form of $u^{i j} K L$ and $v^{i j K L}$ for the $\mathrm{SO}(7)^{ \pm}$invariant solutions, given in appendix A, and the following identifies satisfied by $C_{ \pm}^{I J K L}[27,34]$

$$
\begin{aligned}
& C_{+}^{I J M N} C_{+}^{M N K L}=12 \delta_{K L}^{I J}+4 C_{+}^{I J K L}, \\
& C_{-}^{I J M N} C_{-}^{M N K L}=12 \delta_{K L}^{I J}-4 C_{-}^{I J K L},
\end{aligned}
$$

the above equation reduces to

$$
\left(u_{i j}^{I J}+v_{i j I J}\right)\left(u_{K L}^{i j}+v^{i j K L}\right)=\left(c^{3}+\epsilon s^{3}\right) \delta_{K L}^{I J}+\frac{1}{2} \epsilon c s(c+s) C_{+}^{I J K L}-\frac{1}{2}(1-\epsilon) c s^{2} C_{-}^{I J K L},
$$

where

$$
\epsilon= \begin{cases}1 & \mathrm{SO}(7)^{+} \\ 0 & \mathrm{SO}(7)^{-}\end{cases}
$$

Dualising $\Gamma_{m_{1} \ldots m_{5}}$ and using the identities satisfied by the contraction of $C_{ \pm}^{I J K L}$ with $K^{m I J}$ and $K^{m n I J}$ (see Ref. [3]) gives

$$
\begin{aligned}
& A_{n m_{1} \ldots m_{5}}-\frac{\sqrt{2}}{4} A_{n\left[m_{1} m_{2}\right.} A_{\left.m_{3} m_{4} m_{5}\right]} \\
& =\frac{\sqrt{2}}{4 \cdot 6 !} \Delta g_{n p}\left\{12 \cdot 6 !\left(c^{3}+\epsilon s^{3}\right) \stackrel{\circ}{\zeta}_{m_{1} \ldots m_{5}}^{p}\right. \\
& -\frac{c s(c+s)}{3} \epsilon\left[{\stackrel{\circ}{m_{1} \ldots m_{5}}}_{q}^{p} \xi^{q}+6 \cdot 6 !{\stackrel{\circ}{\zeta} m_{1} \ldots m_{5} q}^{q}\left((3+\xi) \stackrel{\circ}{g}^{p q}-(21+\xi) \hat{\xi}^{m} \hat{\xi}^{n}\right)\right] \\
& \left.-(1-\epsilon) c s^{2} \stackrel{\circ}{\eta}_{m_{1} \ldots m_{5} r s} \stackrel{\circ}{S}^{p r s}\right\} \text {. }
\end{aligned}
$$

To compare this result, obtained from the uplift formula, with the results directly obtained by solving the $D=11$ field equations, let us first consider the $\mathrm{SO}(7)^{+}$invariant solution. Using

$$
c s(c+s)=\gamma^{1 / 2} / 5, \quad c^{3}+s^{3}=2 \gamma^{1 / 2} / 5
$$


and noting that $A_{m n p}=0$ for this solution, equation (103) reduces to

$$
A_{m_{1} \ldots m_{6}}=\frac{\sqrt{2}}{2 \cdot 6 !} \frac{1}{9-\xi} \stackrel{\circ}{\eta}_{m_{1} \ldots m_{6} p} \xi^{p}-3 \sqrt{2} \stackrel{\circ}{\zeta}_{m_{1} \ldots m_{6}}
$$

where we have used the form of the metric $g_{m n}$ given in equation (116). Now, taking the exterior derivative of this equation gives

$$
7 ! \stackrel{\circ}{D}_{\left[m_{1}\right.} A_{\left.m_{2} \ldots m_{7}\right]}=-\frac{180 \sqrt{2}}{(9-\xi)^{2}} m_{7} \stackrel{\circ}{\eta}_{m_{1} \ldots m_{7}}
$$

which agrees precisely with the expression found by dualising the Freund-Rubin field strength for the value of $\gamma$ set by the non-linear ansätze, see equation (120).

Next, consider the $\mathrm{SO}(7)^{-}$invariant solution. Using the expressions for $A_{m n p}$ and $g_{m n}$ in appendix $\mathrm{A}$, it is simple to show that equation (103) reduces to

$$
A_{m_{1} \ldots m_{6}}=-3 \sqrt{2} \stackrel{\circ}{\zeta}_{m_{1} \ldots m_{6}}+\frac{\sqrt{2}}{16 \cdot 6 !} \gamma^{-1 / 3} \stackrel{\circ}{S}_{\left[m_{1}\right.}^{p q} \stackrel{\circ}{\eta}_{\left.m_{2} \ldots m_{6}\right] p q}
$$

However, the second term on the right hand side vanishes by the Schouten identity and the tracelessness of the torsion. Hence,

$$
A_{m_{1} \ldots m_{6}}=-3 \sqrt{2} \dot{\zeta}_{m_{1} \ldots m_{6}},
$$

which agrees with the expression for $A_{m_{1} \ldots m_{6}}$ given in equation (130) for the particular value of $\gamma$ set by the ansätze.

\section{Outlook: magnetic vectors and the embedding tensor}

Having established the full consistency of all equations for the $S^{7}$ compactification we now return to the most remarkable feature of the vielbein postulate equations (24)-(27), namely the fact that they simultaneously involve the Kaluza-Klein vectors, the 'non-geometric vectors' coming from the threeform field, and the $D=11$ dual vector fields. In principle, it is therefore clear that both 'electric' vector fields coming from the reduction of $B_{\mu}{ }^{m}$ and $B_{\mu m_{1} \ldots m_{5}}$, and 'magnetic' vector fields, coming from the reduction of $B_{\mu m n}$, can be gauged. This is a feature that our construction shares with the embedding tensor formalism as applied to gaugings of maximal supergravity in four dimensions [10, 11, 12. There as well, one initially works with the full set of 56 electric and magnetic vector fields, replacing the Cartan equations (58) with the more general ansatz

$$
\mathcal{V}^{-1}(x)\left[\partial_{\mu}-g A_{\mu}^{I J}(x) \Theta_{I J} \mathcal{A} \mathcal{Y}^{\mathcal{A}}-g A_{\mu I J}(x) \Theta^{I J}{ }_{\mathcal{A}} \mathcal{Y}^{\mathcal{A}}\right] \mathcal{V}(x)=Q_{\mu}(x)+P_{\mu}(x),
$$

where $\mathcal{Y}^{\mathcal{A}}(\mathcal{A}=1, \ldots, 133)$ are the generators of $\mathrm{E}_{7(7)},\left(\Theta_{I J \mathcal{A}}, \Theta^{I J}{ }_{\mathcal{A}}\right)$ is the embedding tensor, and $A_{\mu}^{I J}$ and $A_{\mu I J}$ are the electric and magnetic vectors introduced in (37) and (38), respectively. The embedding tensor thus transforms in the product $\mathbf{5 6} \otimes \mathbf{1 3 3}$, but a consistent gauging with 28 propagating gauge fields exists only when $\Theta$ restricts to the $\mathbf{9 1 2}$ representation of $\mathrm{E}_{7(7)}$ in this product [10, 11, 12] (and in addition satisfies a quadratic identity). The choice of embedding tensor not only determines the gauge group, but also decides which 28 vector fields out of the initial 56 vectors become propagating non-abelian vectors. Consequently, studying the vielbein equations (24) - (27) in parallel with (109) should thus enable one to understand the embedding tensor and its relation to any particular compactification directly from the eleven-dimensional perspective. Although we will leave the full exploration of these possibilities to future work, we conclude with some comments. 
In the $S^{7}$ compactification considered in section 4, the $\mathrm{SO}(8)$ gauge covariant derivative term comes from the following terms in the generalised vielbein postulates:

$$
\partial_{m} B_{\mu}^{n} \quad \text { and } \quad \partial_{\left[m_{1}\right.} B_{\left.|\mu| m_{2} \ldots m_{6}\right]}
$$

In particular, terms of the form

$$
\partial_{m} B_{\mu}{ }^{m} \quad \text { and } \quad \partial_{[m} B_{|\mu| n p]}
$$

do not contribute. The first expression above vanishes because the Kaluza-Klein ansatz for the graviphoton is given by $S^{7}$ Killing vectors, which are divergence-free; while the second expression vanishes because of the form of the Kaluza-Klein ansatz for $B_{\mu m n}$, equation (40), and properties of $S^{7}$ Killing spinors.

A natural question to ask is whether one can find examples of compactifications where the expressions (111), that vanish for the $S^{7}$ reduction, contribute to the four-dimensional Cartan equation (109). For example, while the first expression vanishes for Killing vectors, it is non-zero for conformal Killing vectors, which also form a simple Lie algebra. An interesting question is whether one can carry out more general reductions of this type.

Furthermore, a particularly interesting class of gaugings to investigate in this context are the Scherk-Schwarz compactifications [35] and twisted 7-torus flux compactifications [36, 37, 38, 39, 40], which lead to various gaugings in four dimensions (see 12] for a review of known gaugings in four dimensions). While the original Scherk-Schwarz reductions on flat groups are known to lead to electric gaugings [41, flux compactifications provide examples where both electric and magnetic vector fields contribute to the gauging [38].

A study of the generalised vielbein postulates may also shed light on the higher-dimensional origins of the recently discovered continuous family of inequivalent maximal $\mathrm{SO}(8)$ gauged supergravities [30. While the original $\mathrm{SO}(8)$ gauged supergravity $[8$ in the $\mathrm{SL}(8, \mathbb{R})$ symplectic frame only contains electric gaugings, there is a deformation that allows both electric and magnetic gaugings in the aforementioned symplectic frame. For a given range of the angle of rotation between gaugings of electric and magnetic vector fields the theory is inequivalent to the original theory. While $D=11$ supergravity apparently cannot explain the existence of these new supergravities, with the framework presented here it is possible to investigate whether $D=11$ supergravity admits an analogous deformation that rotates $B_{\mu}{ }^{\mathrm{MN}}$ and $B_{\mu \mathrm{MN}}$, defined in (20), into each other and that would be implemented by a rotation on the 56-bein (15) in complete analogy with the $D=4$ theory [2]. The $S^{7}$ compactification of these putative theories would then give rise to the magnetic gaugings in the deformed theories found in [30]. 


\section{A $\quad \operatorname{SO}(7)^{ \pm}$invariant solutions}

In this appendix, we summarise the $\mathrm{SO}(7)^{ \pm}$invariant stationary points of maximal gauged supergravity [29] and their respective eleven-dimensional counterpart solutions [27, 28]. The non-linear metric and flux ansätze have been confirmed for these solutions in Ref. [26, 3]. Much of the necessary information regarding these solutions is explained in Ref. [3] and in particular its appendix A. Therefore, for brevity, we refer the reader there for the definitions of the relevant structures and content ourselves here with a list of the most important properties of these solutions that will be relevant for the calculations in section 5 .

The scalar profile for the $\mathrm{SO}(7)^{+}$invariant stationary point is given by [34, 26 ]

$$
\begin{aligned}
& u_{K L}^{I J}=p^{3} \delta_{K L}^{I J}+\frac{1}{2} p q^{2} C_{+}^{I J K L}, \\
& v^{I J K L}=q^{3} \delta_{K L}^{I J}+\frac{1}{2} p^{2} q C_{+}^{I J K L},
\end{aligned}
$$

where constants $p$ and $q$ are such that 29

$$
\begin{aligned}
& c^{2}=\left(p^{2}+q^{2}\right)^{2}=\frac{1}{2}(3 / \sqrt{5}+1), \\
& s^{2}=(2 p q)^{2}=\frac{1}{2}(3 / \sqrt{5}-1) .
\end{aligned}
$$

The eleven-dimensional solution is of the form [27]

$$
\begin{aligned}
g_{M N} & =\gamma^{7 / 18} 30^{-2 / 3}(9-\xi)^{2 / 3}\left(\stackrel{\circ}{\eta}_{\mu \nu}, \frac{\gamma^{-1 / 2}}{9-\xi}\left[30 \stackrel{\circ}{m}_{m n}-(21+\xi) \hat{\xi}_{m} \hat{\xi}_{n}\right]\right), \\
F_{M N P Q} & =\left(\frac{\sqrt{6}}{3} i m_{7} \gamma^{5 / 6} \stackrel{\circ}{\eta \nu \rho \sigma}_{\mu \nu}, 0\right),
\end{aligned}
$$

where $\gamma$ is an arbitrary constant, which takes the value

$$
\gamma=5^{3 / 2}
$$

when the solution is constructed via the non-linear ansätze [3]. Note that the determinant of the siebenbein

$$
\Delta=\operatorname{det}\left(e_{m}{ }^{a}\right)=\sqrt{\operatorname{det}\left(g_{m n}\right)}=\gamma^{-7 / 18} 30^{2 / 3}(9-\xi)^{-2 / 3} .
$$

In addition, due to the existence of the Freund-Rubin term, the dual potential $A_{(6)}$ is non-zero and of the form

$$
7 ! D_{\left[M_{1}\right.} A_{\left.M_{2} \ldots M_{7}\right]}=\left\{\begin{array}{ll}
-180 \sqrt{10} m_{7} \gamma^{-1 / 3}(9-\xi)^{-2}{\stackrel{\circ}{\eta}}_{m_{1} \ldots m_{7}} & {\left[m_{1} \ldots m_{7}\right]} \\
0 & \text { otherwise }
\end{array} .\right.
$$

The scalar profile of the $\mathrm{SO}(7)^{-}$invariant stationary point of maximal supergravity is of the form 34, 26]

$$
\begin{gathered}
u_{K L}^{I J}=p^{3} \delta_{K L}^{I J}-\frac{1}{2} p q^{2} C_{-}^{I J K L}, \\
v^{I J K L}=i q^{3} \delta_{K L}^{I J}-\frac{1}{2} i p^{2} q C_{-}^{I J K L},
\end{gathered}
$$


where constants $c$ and $s$, related to $p$ and $q$, as above take the values [29]

$$
c^{2}=\frac{5}{4}, \quad s^{2}=\frac{1}{4}
$$

The eleven-dimensional solution is of the form 28

$$
\begin{aligned}
g_{M N} & =\gamma^{7 / 18}\left(\stackrel{\circ}{\eta}_{\mu \nu}, \gamma^{-1 / 2} \stackrel{\circ}{g}_{m n}\right) \\
F_{M N P Q} & =\left(2 \sqrt{2} i m_{7} \gamma^{5 / 6} \stackrel{\circ}{\eta}_{\mu \nu \rho \sigma}, \frac{\sqrt{2}}{6} m_{7} \gamma^{-1 / 6} \stackrel{\circ}{\eta}_{m n p q r s t} \stackrel{\circ}{S}^{r s t}\right),
\end{aligned}
$$

and in particular,

$$
A_{M N P}= \begin{cases}2 \sqrt{2} i \gamma^{5 / 6} \stackrel{\circ}{\zeta}_{\mu \nu \rho} & {[\mu \nu \rho]} \\ \frac{\sqrt{2}}{4 !} \gamma^{-1 / 6} \stackrel{\circ}{S}_{m n p} & {[m n p]} \\ 0 & \text { otherwise }\end{cases}
$$

where $\stackrel{\circ}{\zeta}_{\mu \nu \rho}$ is the potential for the Freund-Rubin field strength

$$
4 ! \partial_{[\mu} \stackrel{\circ}{\zeta}_{\nu \rho \sigma]}=m_{7} \stackrel{\circ}{\eta}_{\mu \nu \rho \sigma}
$$

As before, $\gamma$ is an arbitrary constant that is fixed by the non-linear ansätze to take the value [3]

$$
\gamma^{1 / 3}=5 / 4
$$

Furthermore,

$$
\Delta=\operatorname{det}\left(e_{m}^{a}\right)=\sqrt{\operatorname{det}\left(g_{m n}\right)}=\gamma^{-7 / 18} .
$$

The six-form potential for this solution is of the form [4]

$$
A_{M_{1} \ldots M_{6}}= \begin{cases}\frac{\sqrt{2}}{12} i \gamma^{2 / 3} \stackrel{\circ}{\zeta \nu \rho}_{\mu} \stackrel{\circ}{S}_{m n p} & {[\mu \nu \rho m n p]} \\ -\frac{15 \sqrt{2}}{4} \gamma^{-1 / 3} \stackrel{\circ}{\zeta}_{m_{1} \ldots m_{6}} & {\left[m_{1} \ldots m_{6}\right]} \\ 0 & \text { otherwise }\end{cases}
$$

where $\stackrel{\circ}{\zeta}_{m_{1} \ldots m_{6}}$ is defined in equation (48).

\section{B Useful identities}

We list some useful identities satisfied by 7 -dimensional $\Gamma$-matrices. These identities already appear in Refs. [14] and [1].

$$
\begin{aligned}
& \Gamma^{a_{1} \ldots a_{7}}=-i \epsilon^{a_{1} \ldots a_{7}}, \\
& \Gamma^{a_{1} \ldots a_{6}}=-i \epsilon^{a_{1} \ldots a_{6} b} \Gamma^{b}, \\
& \Gamma^{a_{1} \ldots a_{5}}=\frac{i}{2} \epsilon^{a_{1} \ldots a_{5} b c} \Gamma^{b c}, \\
& \Gamma^{a_{1} \ldots a_{4}}=\frac{i}{3 !} \epsilon^{a_{1} \ldots a_{4} b c d} \Gamma^{b c d}, \\
& \Gamma_{A B}^{a b} \Gamma_{C D}^{b}-\Gamma_{A B}^{b} \Gamma_{C D}^{a b}=8 \delta_{[C \mid[A} \Gamma_{B] \mid D]}^{a}, \\
& \Gamma_{A B}^{c[a} \Gamma_{C D}^{b] c}+\Gamma_{A B}^{[a} \Gamma_{C D}^{b]}=4 \delta_{[C \mid[A} \Gamma_{B] \mid D]}^{a b} .
\end{aligned}
$$

These identities are exactly identities (A.1), (A.6) and (A.7) in the appendix A of [1]. 


\section{References}

[1] B. de Wit and H. Nicolai, "d = 11 supergravity with local SU(8) invariance," Nucl.Phys. B274 (1986) 363.

[2] B. de Wit and H. Nicolai, "Deformations of gauged $\mathrm{SO}(8)$ supergravity and supergravity in eleven dimensions," JHEP 1305 (2013) 077, arXiv:1302.6219 [hep-th].

[3] H. Godazgar, M. Godazgar, and H. Nicolai, "Testing the non-linear flux ansatz for maximal supergravity," Phys.Rev. D87 (2013) 085038, arXiv:1303.1013 [hep-th].

[4] H. Godazgar, M. Godazgar, and H. Nicolai, "Generalised geometry from the ground up," arXiv:1307.8295 [hep-th].

[5] E. Cremmer, B. Julia, and J. Scherk, "Supergravity theory in eleven-dimensions," Phys.Lett. B76 (1978) 409-412.

[6] P. G. Freund and M. A. Rubin, "Dynamics of dimensional reduction," Phys.Lett. B97 (1980) 233-235.

[7] M. J. Duff and C. N. Pope, "Kaluza-Klein supergravity and the seven sphere," in Supersymmetry and supergravity 82, S. Ferrara, J. G. Taylor, and P. van Nieuwenhuizen, eds. World Scientific, 1983.

[8] B. de Wit and H. Nicolai, "N=8 supergravity," Nucl.Phys. B208 (1982) 323.

[9] H. Nicolai and H. Samtleben, "Maximal gauged supergravity in three-dimensions," Phys.Rev.Lett. 86 (2001) 1686-1689, arXiv:hep-th/0010076 [hep-th].

[10] H. Nicolai and H. Samtleben, "Compact and noncompact gauged maximal supergravities in three-dimensions," JHEP 0104 (2001) 022, arXiv:hep-th/0103032 [hep-th].

[11] B. de Wit, H. Samtleben, and M. Trigiante, "On Lagrangians and gaugings of maximal supergravities," Nucl.Phys. B655 (2003) 93-126, arXiv:hep-th/0212239 [hep-th].

[12] B. de Wit, H. Samtleben, and M. Trigiante, "The Maximal D=4 supergravities," JHEP 0706 (2007) 049, arXiv:0705.2101 [hep-th].

[13] E. Cremmer and B. Julia, "The N=8 supergravity theory. 1. The Lagrangian," Phys.Lett. B80 (1978) 48 .

[14] E. Cremmer and B. Julia, "The SO(8) Supergravity," Nucl.Phys. B159 (1979) 141.

[15] A. Coimbra, C. Strickland-Constable, and D. Waldram, "Supergravity as generalised geometry II: $e_{d(d)} \times \mathbb{R}^{+}$and M theory," arXiv:1212.1586 [hep-th].

[16] J.-H. Park and Y. Suh, "U-geometry : SL(5)," JHEP 04 (2013) 147, arXiv:1302.1652 [hep-th].

[17] G. Aldazabal, M. Graña, D. Marqus, and J. Rosabal, "Extended geometry and gauged maximal supergravity," JHEP 1306 (2013) 046, arXiv:1302.5419 [hep-th].

[18] M. Cederwall, J. Edlund, and A. Karlsson, "Exceptional geometry and tensor fields," JHEP 1307 (2013) 028, arXiv:1302.6736 [hep-th]. 
[19] H. Godazgar, M. Godazgar, and M. J. Perry, "E8 duality and dual gravity," JHEP 1306 (2013) 044, arXiv: 1303.2035 [hep-th].

[20] M. Hatsuda and K. Kamimura, "M5 algebra and SO(5,5) duality," JHEP 1306 (2013) 095, arXiv:1305.2258 [hep-th].

[21] D. S. Berman and D. C. Thompson, "Duality symmetric string and M-theory," arXiv:1306.2643 [hep-th].

[22] O. Hohm and H. Samtleben, "Exceptional form of D=11 supergravity," arXiv:1308.1673 [hep-th].

[23] R. Kerner, "Generalization of the Kaluza-Klein theory for an arbitrary nonabelian gauge group," Annales Poincare Phys. Theor. 9 (1968) 143-152.

[24] Y. Cho and P. G. Freund, "Nonabelian gauge fields in Nambu-Goldstone fields," Phys.Rev. D12 (1975) 1711.

[25] A. Salam and J. Strathdee, "On Kaluza-Klein theory," Annals Phys. 141 (1982) 316-352.

[26] B. de Wit, H. Nicolai, and N. P. Warner, "The embedding of gauged N=8 supergravity into d = 11 supergravity," Nucl.Phys. B255 (1985) 29.

[27] B. de Wit and H. Nicolai, "A new $\mathrm{SO}(7)$ invariant solution of $\mathrm{d}=11$ supergravity," Phys.Lett. B148 (1984) 60.

[28] F. Englert, "Spontaneous compactification of eleven-dimensional supergravity," Phys.Lett. B119 (1982) 339.

[29] N. Warner, "Some new extrema of the scalar potential of gauged N=8 supergravity," Phys.Lett. B128 (1983) 169.

[30] G. Dall'Agata, G. Inverso, and M. Trigiante, "Evidence for a family of SO(8) gauged supergravity theories," Phys.Rev.Lett. 109 (2012) 201301, arXiv:1209.0760 [hep-th].

[31] C. Hillmann, "Generalized E(7(7)) coset dynamics and D=11 supergravity," JHEP 0903 (2009) 135, arXiv:0901.1581 [hep-th].

[32] B. de Wit and H. Nicolai, "The consistency of the $\mathrm{S}^{* *} 7$ truncation in $\mathrm{D}=11$ supergravity," Nucl.Phys. B281 (1987) 211.

[33] H. Nicolai and K. Pilch, "Consistent truncation of d $=11$ supergravity on $\operatorname{AdS}_{4} \times S^{7}$," JHEP 1203 (2012) 099, arXiv:1112.6131 [hep-th].

[34] B. de Wit and H. Nicolai, "The parallelizing S(7) torsion in gauged N=8 supergravity," Nucl.Phys. B231 (1984) 506.

[35] J. Scherk and J. H. Schwarz, "How to Get Masses from Extra Dimensions," Nucl.Phys. B153 (1979) 61-88.

[36] G. Dall'Agata and S. Ferrara, "Gauged supergravity algebras from twisted tori compactifications with fluxes," Nucl.Phys. B717 (2005) 223-245,

arXiv:hep-th/0502066 [hep-th]. 
[37] L. Andrianopoli, M. Lledo, and M. Trigiante, "The Scherk-Schwarz mechanism as a flux compactification with internal torsion," JHEP 0505 (2005) 051,

arXiv:hep-th/0502083 [hep-th].

[38] R. D'Auria, S. Ferrara, and M. Trigiante, "E(7(7)) symmetry and dual gauge algebra of M-theory on a twisted seven-torus," Nucl.Phys. B732 (2006) 389-400, arXiv:hep-th/0504108 [hep-th].

[39] R. D'Auria, S. Ferrara, and M. Trigiante, "Curvatures and potential of M-theory in D=4 with fluxes and twist," JHEP 0509 (2005) 035, arXiv:hep-th/0507225 [hep-th].

[40] C. Hull and R. Reid-Edwards, "Flux compactifications of M-theory on twisted Tori," JHEP 0610 (2006) 086, arXiv:hep-th/0603094 [hep-th].

[41] L. Andrianopoli, R. D'Auria, S. Ferrara, and M. Lledo, "Gauging of flat groups in four-dimensional supergravity," JHEP 0207 (2002) 010, arXiv:hep-th/0203206 [hep-th]. 